Submitted to the Annals of Statistics

arXiv: arXiv: 1501.00312

\title{
SUPPLEMENT TO: STATISTICAL CONSISTENCY AND ASYMPTOTIC NORMALITY FOR HIGH-DIMENSIONAL ROBUST $M$-ESTIMATORS
}

\author{
By Po-Ling LOH \\ University of Wisconsin - Madison

\begin{abstract}
This supplement contains supporting appendices for the main manuscript, "Statistical consistency and asymptotic normality for high-dimensional robust $M$-estimators."
\end{abstract}

\section{APPENDIX A: MEASURES OF ROBUSTNESS}

Various methods exist in the classical literature for quantifying the robustness of statistical estimation procedures. In this section, we provide a review of breakdown points, influence functions, and asymptotic variance of robust estimators, and cite relevant literature. by

The finite-sample breakdown point of an estimator $T_{n}$ on the sample $\left\{x_{i}\right\}_{i=1}^{n}$ is defined

$$
F B P_{n}\left(T ; x_{1}, \ldots, x_{n}\right):=\frac{1}{n} \cdot \min \left\{m: \max _{i_{1}, \ldots, i_{m}} \sup _{y_{1}, \ldots, y_{m}}\left|T_{n}\left(z_{1}, \ldots, z_{n}\right)\right|=\infty\right\}
$$

where $\left(z_{1}, \ldots, z_{n}\right)$ is the sample obtained from $\left(x_{1}, \ldots, x_{n}\right)$ by replacing the data points $\left(x_{i_{1}}, \ldots, x_{i_{m}}\right)$ by $\left(y_{1}, \ldots, y_{m}\right)$ (Hampel et al., 2011; Donoho and Huber, 1983). One may verify that the finite-sample breakdown point is $\frac{1}{n}$ for $M$-estimators of the type defined in Section 2.1 when $\ell$ is convex (Alfons, Croux and Gelper, 2013). This provides another reason to use nonconvex loss functions in order to obtain a robust estimator. Although the breakdown behavior of $M$-estimators is much harder to characterize when the loss function is nonconvex, Maronna, Bustos and Yohai (1979) derived theoretical results showing the breakdown point decays as $\mathcal{O}\left(p^{-1 / 2}\right)$ when the $x_{i}$ 's are Gaussian. More recently, Wang et al. (2013) analyzed the breakdown point of a certain nonconvex penalized $M$-estimator, but their analysis is again very specific to the precise form of the estimator and requires careful data-dependent tuning of the scale parameter used in the objective function. Under suitable regularity conditions, taking the limit of the finite breakdown point as $n \rightarrow \infty$ yields the asymptotic breakdown point, but the latter concept is more technical and we do not discuss it here.

A second measure of robustness is given by the influence function. At the population level, the influence function of an estimator $T$ on a distribution $F$ with respect to a point 
$(x, y)$ is defined by

$$
I F((x, y) ; T, F)=\lim _{t \rightarrow 0^{+}} \frac{T\left((1-t) F+t \delta_{(x, y)}\right)-T(F)}{t},
$$

where $\delta_{(x, y)}$ is a point mass at $(x, y)$. The gross error sensitivity is defined in terms of the influence function as

$$
G E S(T, F):=\sup _{(x, y)}|I F((x, y) ; T, F)|,
$$

and the estimator $T$ is $B$-robust if $G E S(T, F)<\infty$ (Rousseeuw, 1981). In the linear regression case, let $F_{\beta}$ denote the distribution on $\left(x_{i}, y_{i}, \epsilon_{i}\right)$ parametrized by $\beta$. If $T_{\ell}$ minimizes the $M$-estimator defined in equation (4) and $\ell$ is twice differentiable, the influence function takes the form

$$
I F\left((x, y) ; T_{\ell}, F_{\beta}\right)=\ell^{\prime}\left(x^{T} \beta-y\right) \cdot\left(\mathbb{E}\left[\ell^{\prime \prime}\left(x_{i}^{T} \beta-y_{i}\right) \cdot x_{i} x_{i}^{T}\right]\right)^{-1} x,
$$

where the expectation is taken with respect to $F_{\beta}$ (Hampel et al., 2011, Section 6.3). In particular, if the $x_{i}$ 's are fixed and contamination is only allowed in the $y_{i}$ 's, the influence function in equation (26) is bounded as a function of $y$, provided $\ell^{\prime}$ is bounded. For a generalized $M$-estimator $T_{\eta}$ defined by equation (7), the influence function is given by

$$
\operatorname{IF}\left((x, y) ; T_{\eta}, F_{\beta}\right)=\eta\left(x, x^{T} \beta-y\right) \cdot\left(\mathbb{E}\left[\left.\left(\frac{\partial \eta(x, r)}{\partial r}\right)\right|_{\left(x_{i}, y_{i}\right)} \cdot x_{i} x_{i}^{T}\right]\right)^{-1} x
$$

where the expectation is taken with respect to $F_{\beta}$ (Hampel et al., 2011). In particular, if $\eta$ takes the form in equation (8), then equation (27) simplifies to

$$
I F\left((x, y) ; T_{\eta}, F_{\beta}\right)=w(x) \ell^{\prime}\left(\left(x^{T} \beta-y\right) v(x)\right) \cdot\left(\mathbb{E}\left[w\left(x_{i}\right) v\left(x_{i}\right) \cdot \ell^{\prime \prime}\left(r_{i} v\left(x_{i}\right)\right) \cdot x_{i} x_{i}^{T}\right]\right)^{-1} x,
$$

and we see that the overall influence function is bounded whenever $\ell^{\prime}$ is bounded and $w$ is defined in such a way that $\|w(x) x\|_{2}$ is bounded.

A finite-sample version of the influence function is known as the sensitivity curve, and under suitable regularity conditions, the sensitivity curve converges to the influence function as $n \rightarrow \infty$ (Hampel et al., 2011). The literature concerning influence functions for high-dimensional estimators is again rather sparse, but has been a topic of recent interest (Avella Medina, 2014; Öllerer, Croux and Alfons, 2014).

Finally, we turn to second-order considerations. In the classical low-dimensional setting when $p$ is fixed and $n \rightarrow \infty$, Maronna and Yohai (1981) show that under appropriate regularity conditions, the asymptotic variance of an $M$-estimator is given by

$$
V(T, F)=\int I F((x, y) ; T, F) \cdot I F((x, y) ; T, F) d F(x, y) .
$$


By the celebrated Cramér-Rao bound (Lehmann and Casella, 1998), when the $x_{i}$ 's are fixed and the $\epsilon_{i}$ 's are i.i.d., the asymptotic variance $V(T, F)$ of any unbiased estimator is bounded below by the inverse of the Fisher information of the underlying distribution. Furthermore, this lower bound is achieved when $T$ is the MLE, in which case $T$ is also asymptotically normally distributed (Godambe, 1960; Lehmann and Casella, 1998). As pointed out in the previous paragraph, however, the influence function of the MLE may not be bounded, leading to a critical tradeoff in designing robust $M$-estimators. In addition, the behavior of the asymptotic variance is much harder to analyze when both $n$ and $p$ are allowed to grow. Several recent papers (El Karoui et al., 2013; Bean et al., 2013; Donoho and Montanari, 2013) examine the setting where $\frac{n}{p} \rightarrow \delta \in(1, \delta)$, and show that the asymptotic variance of the (unregularized) $M$-estimator coming from a convex loss function includes an additional term not present in the classical fixed- $p$ case. In contrast, we show that with the proper choice of nonconvex penalty, local solutions of nonconvex regularized $M$-estimators coincide with the oracle solution, so they inherit certain optimality properties from classical robust estimation theory. It is these higher-order considerations that reveal the true advantage of using nonconvex loss functions for robust $M$-estimation; although estimators such as the LAD Lasso (Wang, Li and Jiang, 2007; Wang, 2013) may also be shown to be statistically consistent under reasonable assumptions, the LAD loss is a suboptimal choice from the viewpoint of asymptotic efficiency, under the high-dimensional scaling $n \geq C k \log p$ and oracle conditions, unless the additive errors follow a double exponential distribution

\section{APPENDIX B: REVIEW OF LOSSES, PENALTIES, AND WEIGHTING FUNCTIONS}

In this Appendix, we remind the reader of several important loss functions, penalty functions, and weight functions that are referenced throughout the paper.

B.1. Robust loss functions. We begin by defining various loss functions prevalent in the robust statistics literature.

Huber loss. We have

$$
\ell(u)= \begin{cases}\frac{u^{2}}{2}, & \text { if }|u| \leq \xi \\ \xi|u|-\frac{\xi^{2}}{2}, & \text { if }|u|>\xi\end{cases}
$$

In this case, $\ell$ is a convex function. Although $\ell^{\prime \prime}$ does not exist everywhere, $\ell^{\prime}$ is continuous and $\left\|\ell^{\prime}\right\|_{\infty} \leq \xi$.

Tukey's biweight. We have

$$
\ell(u)= \begin{cases}\frac{\xi^{2}}{6}\left(1-\left(1-\frac{u^{2}}{\xi^{2}}\right)^{3}\right), & \text { if }|u| \leq \xi \\ \frac{\xi^{2}}{6}, & \text { if }|u|>\xi\end{cases}
$$


Note that $\ell$ is nonconvex. We also compute the first derivative

$$
\ell^{\prime}(u)= \begin{cases}u\left(1-\frac{u^{2}}{\xi^{2}}\right)^{2}, & \text { if }|u| \leq \xi \\ 0, & \text { if }|u|>\xi\end{cases}
$$

and second derivative

$$
\ell^{\prime \prime}(u)= \begin{cases}\left(1-\frac{u^{2}}{\xi^{2}}\right)\left(1-\frac{5 u^{2}}{\xi^{2}}\right), & \text { if }|u| \leq \xi \\ 0, & \text { if }|u|>\xi\end{cases}
$$

Note that $\ell^{\prime \prime}$ is continuous. Furthermore, $\left\|\ell^{\prime}\right\|_{\infty} \leq \frac{16 \xi}{25 \sqrt{5}}$. One may check that Tukey's biweight function is not an MLE. Furthermore, although $\ell^{\prime \prime}$ exists everywhere and is continuous, $\ell^{\prime \prime \prime}$ does not exist for $u \in\left\{ \pm \xi, \pm \frac{\xi}{\sqrt{5}}\right\}$.

Cauchy loss. We have

$$
\ell(u)=\frac{\xi^{2}}{2} \log \left(1+\frac{u^{2}}{\xi^{2}}\right)
$$

Note that $\ell$ is nonconvex. When $\xi=1$, the function $\ell(u)$ is proportional to the MLE for the $t$-distribution with one degree of freedom (a heavy-tailed distribution). This suggests that for heavy-tailed distributions, nonconvex loss functions may be more desirable from the point of view of statistical efficiency, although optimization becomes more difficult; this idea is explored more fully in Section 3.3. For the Cauchy loss, we have

$$
\ell^{\prime}(u)=\frac{u}{1+u^{2} / \xi^{2}}, \quad \text { and } \quad \ell^{\prime \prime}(u)=\frac{1-u^{2} / \xi^{2}}{\left(1+u^{2} / \xi^{2}\right)^{2}} .
$$

In particular, $\left|\ell^{\prime}(u)\right|$ is maximized when $u^{2}=\xi^{2}$, so $\left\|\ell^{\prime}\right\|_{\infty} \leq \frac{\xi}{2}$. We may also check that $\left\|\ell^{\prime \prime}\right\|_{\infty} \leq 1$ and $\left\|\ell^{\prime \prime \prime}\right\|_{\infty} \leq \frac{3}{2 \xi}$.

B.2. Robust weight functions. We now define several weight functions that are used in robust regression to decrease the effect of outliers in the covariates.

Mallows estimator (Mallows, 1975). We take $v(x) \equiv 1$ and $w(x)$ of the form

$$
w(x)=\min \left\{1, \frac{b}{\|B x\|_{2}}\right\}, \quad \text { or } \quad w(x)=\min \left\{1, \frac{b^{2}}{\|B x\|_{2}^{2}}\right\},
$$

for parameters $b>0$ and $B \in \mathbb{R}^{p \times p}$. Note that $\|w(x) x\|_{2}$ is indeed bounded for a fixed choice of $b$ and $B$, since

$$
\|w(x) x\|_{2} \leq \frac{\|b x\|_{2}}{\|B x\|_{2}} \leq b\left\|B^{-1}\right\|_{2}:=b_{0} .
$$

The Mallows estimator effectively shrinks data points for which $\left\|x_{i}\right\|_{2}$ is large toward an elliptical shell defined by $B$, and likewise pushes small data points closer to the shell. 
Hill-Ryan estimator (Hill, 1977). We take $v(x)=w(x)$, where $w$ is defined such that $\|w(x) x\|_{2}$ is bounded (e.g., equation (29)). In addition to downweighting the influence function similarly to the Mallows estimator, the Hill-Ryan estimator scales the residuals according to the leverage weight of the $x_{i}$ 's.

Schweppe estimator (Merrill and Schweppe, 1971). For a parameter $B \in \mathbb{R}^{p \times p}$, we take $w(x)=\frac{1}{\|B x\|_{2}}$ and $v(x)=\frac{1}{w(x)}$. Like the Mallows estimator, Schweppe's estimator downweights the contribution of data points with high leverage as a summand in the estimating equation (7). If $\ell^{\prime}$ is locally linear around the origin and flattens out for larger values, Schweppe's estimator additionally dampens the effect of a residual $r_{i}$ only when it is large compared to the leverage of $x_{i}$. As discussed in Hampel et al. (2011), Schweppe's estimator is designed to be optimal in terms of a measure of variance robustness, subject to a bound on the influence function.

B.3. Nonconvex penalties. Finally, we define two common nonconvex penalties.

Smoothly clipped absolute deviation (SCAD) penalty. This penalty, due to Fan and Li (2001), takes the form

$$
\rho_{\lambda}(t):= \begin{cases}\lambda|t|, & \text { for }|t| \leq \lambda, \\ -\frac{t^{2}-2 a \lambda|t|+\lambda^{2}}{2(a-1)}, & \text { for } \lambda<|t| \leq a \lambda, \\ \frac{(a+1) \lambda^{2}}{2}, & \text { for }|t|>a \lambda,\end{cases}
$$

where $a>2$ is fixed. The SCAD penalty is $(\mu, \gamma)$-amenable, with $\mu=\frac{1}{a-1}$ and $\gamma=a$.

Minimax concave penalty (MCP). This penalty, due to Zhang (2010b), takes the form

$$
\rho_{\lambda}(t):=\operatorname{sign}(t) \lambda \cdot \int_{0}^{|t|}\left(1-\frac{z}{\lambda b}\right)_{+} d z
$$

where $b>0$ is fixed. The MCP regularizer is $(\mu, \gamma)$-amenable, with $\mu=\frac{1}{b}$ and $\gamma=b$.

Standard $\ell_{1}$-penalty. The $\ell_{1}$-penalty $\rho_{\lambda}(t)=\lambda|t|$ is an example of a regularizer that is 0 -amenable, but not $(0, \gamma)$-amenable, for any $\gamma<\infty$.

\section{APPENDIX C: PROOFS OF MAIN THEOREMS}

In this Appendix, we provide the proofs of the main theorems in the paper. 
C.1. Proof of Theorem 1. We first suppose the existence of stationary points in the local region; we will establish that fact at the end of the proof. Suppose $\widetilde{\beta}$ is a stationary point such that $\left\|\widetilde{\beta}-\beta^{*}\right\|_{2} \leq r$. Since $\widetilde{\beta}$ is a stationary point and $\beta^{*}$ is feasible, we have the inequality

$$
\left\langle\nabla \mathcal{L}_{n}(\widetilde{\beta})-\nabla q_{\lambda}(\widetilde{\beta})+\lambda \operatorname{sign}(\widetilde{\beta}), \beta^{*}-\widetilde{\beta}\right\rangle \geq 0 .
$$

By the convexity of $\frac{\mu}{2}\|\beta\|_{2}^{2}-q_{\lambda}(\beta)$, we have

$$
\left\langle\nabla q_{\lambda}(\widetilde{\beta}), \beta^{*}-\widetilde{\beta}\right\rangle \geq q_{\lambda}\left(\beta^{*}\right)-q_{\lambda}(\widetilde{\beta})-\frac{\mu}{2}\left\|\widetilde{\beta}-\beta^{*}\right\|_{2}^{2},
$$

so together with inequality (32), we have

$$
\left\langle\nabla \mathcal{L}_{n}(\widetilde{\beta})+\lambda \operatorname{sign}(\widetilde{\beta}), \beta^{*}-\widetilde{\beta}\right\rangle \geq q_{\lambda}\left(\beta^{*}\right)-q_{\lambda}(\widetilde{\beta})-\frac{\mu}{2}\left\|\widetilde{\beta}-\beta^{*}\right\|_{2}^{2}
$$

Since $\left\langle\operatorname{sign}(\widetilde{\beta}), \beta^{*}-\widetilde{\beta}\right\rangle \leq\left\|\beta^{*}\right\|_{1}-\|\widetilde{\beta}\|_{1}$, this means

$$
\left\langle\nabla \mathcal{L}_{n}(\widetilde{\beta}), \beta^{*}-\widetilde{\beta}\right\rangle \geq \rho_{\lambda}(\widetilde{\beta})-\rho_{\lambda}\left(\beta^{*}\right)-\frac{\mu}{2}\left\|\widetilde{\beta}-\beta^{*}\right\|_{2}^{2}
$$

Now denote $\widetilde{\nu}:=\widetilde{\beta}-\beta^{*}$. From the RSC inequality (10), we have

$$
\left\langle\nabla \mathcal{L}_{n}(\widetilde{\beta})-\nabla \mathcal{L}_{n}\left(\beta^{*}\right), \widetilde{\beta}-\beta^{*}\right\rangle \geq \alpha\|\widetilde{\nu}\|_{2}^{2}-\tau \frac{\log p}{n}\|\widetilde{\nu}\|_{1}^{2} .
$$

Combining inequality (35) with inequality (34), we then have

$$
\left(\alpha-\frac{\mu}{2}\right)\|\widetilde{\nu}\|_{2}^{2}-\tau \frac{\log p}{n}\|\widetilde{\nu}\|_{1}^{2}+\left(\rho_{\lambda}(\widetilde{\beta})-\rho_{\lambda}\left(\beta^{*}\right)\right) \leq\left\langle\nabla \mathcal{L}_{n}\left(\beta^{*}\right), \beta^{*}-\widetilde{\beta}\right\rangle,
$$

so by Hölder's inequality, we conclude that

$$
\left(\alpha-\frac{\mu}{2}\right)\|\widetilde{\nu}\|_{2}^{2}-\tau \frac{\log p}{n}\|\widetilde{\nu}\|_{1}^{2}+\left(\rho_{\lambda}(\widetilde{\beta})-\rho_{\lambda}\left(\beta^{*}\right)\right) \leq\left\|\nabla \mathcal{L}_{n}\left(\beta^{*}\right)\right\|_{\infty}\|\widetilde{\nu}\|_{1} .
$$

In particular, under the assumed scaling $\lambda \geq 4\left\|\nabla \mathcal{L}_{n}\left(\beta^{*}\right)\right\|_{\infty}$ and $\lambda \geq 8 \tau R \frac{\log p}{n}$, we have

$$
\begin{aligned}
\left(\alpha-\frac{\mu}{2}\right)\|\widetilde{\nu}\|_{2}^{2} & \leq\left(\rho_{\lambda}\left(\beta^{*}\right)-\rho_{\lambda}(\widetilde{\beta})\right)+\left(2 R \tau \frac{\log p}{n}+\left\|\nabla \mathcal{L}_{n}\left(\beta^{*}\right)\right\|_{\infty}\right)\|\widetilde{\nu}\|_{1} \\
& \leq\left(\rho_{\lambda}\left(\beta^{*}\right)-\rho_{\lambda}(\widetilde{\beta})\right)+\frac{\lambda}{2}\|\widetilde{\nu}\|_{1} \\
& \leq\left(\rho_{\lambda}\left(\beta^{*}\right)-\rho_{\lambda}(\widetilde{\beta})\right)+\frac{1}{2}\left(\rho_{\lambda}(\widetilde{\nu})+\frac{\mu}{2}\|\widetilde{\nu}\|_{2}^{2}\right)
\end{aligned}
$$

implying that

$$
0 \leq\left(\alpha-\frac{3 \mu}{4}\right)\|\widetilde{\nu}\|_{2}^{2} \leq \frac{3}{2} \rho_{\lambda}\left(\beta^{*}\right)-\frac{1}{2} \rho_{\lambda}(\widetilde{\beta})
$$


By Lemma 5 in Loh and Wainwright (2015), we then have

$$
0 \leq 3 \rho_{\lambda}\left(\beta^{*}\right)-\rho_{\lambda}(\widetilde{\beta}) \leq \lambda\left(3\left\|\widetilde{\nu}_{A}\right\|_{1}-\left\|\widetilde{\nu}_{A^{c}}\right\|_{1}\right),
$$

where $A$ is the index set of the $k$ largest elements of $\widetilde{\nu}$ in magnitude. Hence,

$$
\left\|\widetilde{\nu}_{A^{c}}\right\|_{1} \leq 3\left\|\widetilde{\nu}_{A}\right\|_{1}
$$

implying that

$$
\|\widetilde{\nu}\|_{1}=\left\|\widetilde{\nu}_{A}\right\|_{1}+\left\|\widetilde{\nu}_{A^{c}}\right\|_{1} \leq 4\left\|\widetilde{\nu}_{A}\right\|_{1} \leq 4 \sqrt{k}\|\widetilde{\nu}\|_{2}
$$

Combining inequalities (38) and (39) then gives

$$
\left(\alpha-\frac{3 \mu}{4}\right)\|\widetilde{\nu}\|_{2}^{2} \leq \frac{3 \lambda}{2}\left\|\widetilde{\nu}_{A}\right\|_{1}-\frac{\lambda}{2}\left\|\widetilde{\nu}_{A^{c}}\right\|_{1} \leq \frac{3 \lambda}{2}\left\|\widetilde{\nu}_{A}\right\|_{1} \leq 6 \lambda \sqrt{k}\|\widetilde{\nu}\|_{2},
$$

from which we conclude that

$$
\|\widetilde{\nu}\|_{2} \leq \frac{24 \lambda \sqrt{k}}{4 \alpha-3 \mu}
$$

as wanted. Combining the $\ell_{2}$-bound with inequality (40) then yields the $\ell_{1}$-bound.

Finally, in order to establish the existence of stationary points, we simply define $\widehat{\beta} \in \mathbb{R}^{p}$ such that

$$
\widehat{\beta} \in \arg \min _{\left\|\widehat{\beta}-\beta^{*}\right\|_{2} \leq r,\|\widehat{\beta}\|_{1} \leq R}\left\{\mathcal{L}_{n}(\beta)+\rho_{\lambda}(\beta)\right\}
$$

Then $\widehat{\beta}$ is a stationary point of the program (42), so by the argument just provided, we have

$$
\left\|\widehat{\beta}-\beta^{*}\right\|_{2} \leq c \sqrt{\frac{k \log p}{n}} .
$$

Provided $n \geq \frac{C}{r^{2}} \cdot k \log p$, the point $\widehat{\beta}$ will lie in the interior of the sphere of radius $r$ around $\beta^{*}$. Hence, $\widehat{\beta}$ is also a stationary point of the original program (2), guaranteeing the existence of such local stationary points.

C.2. Proof of Theorem 2. This argument is an adaptation of the proofs of Theorems 1 and 2 in the recent paper (Loh and Wainwright, 2014). We follow the primal-dual witness construction introduced there:

(i) Optimize the restricted program

$$
\widehat{\beta}_{S} \in \arg \min _{\beta \in \mathbb{R}^{S}:\|\beta\|_{1} \leq R}\left\{\mathcal{L}_{n}(\beta)+\rho_{\lambda}(\beta)\right\},
$$

and establish that $\left\|\widehat{\beta}_{S}\right\|_{1}<R$. 
(ii) Define $\widehat{z}_{S} \in \partial\left\|\widehat{\beta}_{S}\right\|_{1}$, and choose $\widehat{z}_{S^{c}}$ to satisfy the zero-subgradient condition

$$
\nabla \mathcal{L}_{n}(\widehat{\beta})-\nabla q_{\lambda}(\widehat{\beta})+\lambda \widehat{z}=0,
$$

where $\widehat{z}=\left(\widehat{z}_{S}, \widehat{z}_{S^{c}}\right)$ and $\widehat{\beta}:=\left(\widehat{\beta}_{S}, 0_{S^{c}}\right)$. Show that $\widehat{\beta}_{S}=\widehat{\beta}_{S}^{\mathcal{O}}$ and establish strict dual feasibility: $\left\|\widehat{z}_{S^{c}}\right\|_{\infty}<1$.

(iii) Verify via second-order conditions that $\widehat{\beta}$ is a local minimum of the program (2) and conclude that all stationary points $\widetilde{\beta}$ satisfying $\left\|\widetilde{\beta}-\beta^{*}\right\|_{2} \leq r$ are supported on $S$.

Step (i). By Theorem 1 applied to the restricted program (43), we have

$$
\left\|\widehat{\beta}_{S}-\beta_{S}^{*}\right\|_{1} \leq \frac{80 \lambda k}{2 \alpha-\mu}
$$

so

$$
\left\|\widehat{\beta}_{S}\right\|_{1} \leq\left\|\beta^{*}\right\|_{1}+\left\|\widehat{\beta}_{S}-\beta_{S}^{*}\right\|_{1} \leq \frac{R}{2}+\frac{80 \lambda k}{2 \alpha-\mu}<R,
$$

using the assumptions of the theorem. This establishes step (i) of the PDW construction.

Step (ii). Since $\widehat{\beta}_{S}$ is an interior point of the restricted program (43), it must satisfy a zero-subgradient condition on the restricted program, implying that we may define $\widehat{z}_{S^{c}}$ to satisfy equation (44). We rewrite the zero-subgradient condition (44) as

$$
\left(\nabla \mathcal{L}_{n}(\widehat{\beta})-\nabla \mathcal{L}_{n}\left(\beta^{*}\right)\right)+\left(\nabla \mathcal{L}_{n}\left(\beta^{*}\right)-\nabla q_{\lambda}(\widehat{\beta})\right)+\lambda \widehat{z}=0
$$

and by the fundamental theorem of calculus,

$$
\widehat{Q}\left(\widehat{\beta}-\beta^{*}\right)+\left(\nabla \mathcal{L}_{n}\left(\beta^{*}\right)-\nabla q_{\lambda}(\widehat{\beta})\right)+\lambda \widehat{z}=0,
$$

where $\widehat{Q}:=\int_{0}^{1} \nabla^{2} \mathcal{L}_{n}\left(\beta^{*}+t\left(\widehat{\beta}-\beta^{*}\right)\right) d t$. In block form, this means

$$
\left[\begin{array}{cc}
\widehat{Q}_{S S} & \widehat{Q}_{S S^{c}} \\
\widehat{Q}_{S^{c} S} & \widehat{Q}_{S^{c} S^{c}}
\end{array}\right]\left[\begin{array}{c}
\widehat{\beta}_{S}-\beta_{S}^{*} \\
0
\end{array}\right]+\left[\begin{array}{c}
\nabla \mathcal{L}_{n}\left(\beta^{*}\right)_{S}-\nabla q_{\lambda}\left(\widehat{\beta}_{S}\right) \\
\nabla \mathcal{L}_{n}\left(\beta^{*}\right)_{S^{c}}-\nabla q_{\lambda}\left(\widehat{\beta}_{S^{c}}\right)
\end{array}\right]+\lambda\left[\begin{array}{c}
\widehat{z}_{S} \\
\widehat{z}_{S^{c}}
\end{array}\right]=0 .
$$

We now have the following lemma, concerning the oracle estimator:

Lemma 3. Under the conditions of Theorem 2, we have the bound

$$
\left\|\widehat{\beta}_{S}^{\mathcal{O}}-\beta_{S}^{*}\right\|_{\infty} \leq c \sqrt{\frac{\log k}{n}}
$$

and $\widehat{\beta}_{S}=\widehat{\beta}_{S}^{\mathcal{O}}$. 
Proof. By the optimality of the oracle estimator, we have

$$
\mathcal{L}_{n}\left(\widehat{\beta}^{\mathcal{O}}\right) \leq \mathcal{L}_{n}\left(\beta^{*}\right)
$$

Furthermore, $\mathcal{L}_{n}$ is strongly convex over the restricted region $S_{r}$ by Lemma 1 . Hence,

$$
\mathcal{L}_{n}\left(\beta^{*}\right)+\left\langle\nabla \mathcal{L}_{n}\left(\beta^{*}\right), \widehat{\beta}^{\mathcal{O}}-\beta^{*}\right\rangle+\frac{\alpha}{4}\left\|\widehat{\beta}^{\mathcal{O}}-\beta^{*}\right\|_{2}^{2} \leq \mathcal{L}_{n}\left(\widehat{\beta}^{\mathcal{O}}\right) .
$$

Summing inequalities (46) and (47), we obtain

$$
\begin{aligned}
\frac{\alpha}{4}\left\|\widehat{\beta}^{\mathcal{O}}-\beta^{*}\right\|_{2}^{2} & \leq\left\langle\nabla \mathcal{L}_{n}\left(\beta^{*}\right), \beta^{*}-\widehat{\beta}^{\mathcal{O}}\right\rangle \leq\left\|\nabla \mathcal{L}_{n}\left(\beta^{*}\right)\right\|_{\infty} \cdot\left\|\widehat{\beta}^{\mathcal{O}}-\beta^{*}\right\|_{1} \\
& \leq \sqrt{k}\left\|\nabla \mathcal{L}_{n}\left(\beta^{*}\right)\right\|_{\infty} \cdot\left\|\widehat{\beta}^{\mathcal{O}}-\beta^{*}\right\|_{2},
\end{aligned}
$$

implying that

$$
\left\|\widehat{\beta}^{\mathcal{O}}-\beta^{*}\right\|_{2} \leq \frac{4 \sqrt{k}}{\alpha}\left\|\nabla \mathcal{L}_{n}\left(\beta^{*}\right)\right\|_{\infty}
$$

In particular, when

$$
\frac{4 \sqrt{k}}{\alpha}\left\|\nabla \mathcal{L}_{n}\left(\beta^{*}\right)\right\|_{\infty}<r
$$

the oracle estimator $\widehat{\beta}^{\mathcal{O}}$ is in the interior point of the feasible region, implying in particular that

$$
\left(\nabla \mathcal{L}_{n}\left(\widehat{\beta}^{\mathcal{O}}\right)\right)_{S}=0
$$

Hence, we have

$$
\left(\nabla \mathcal{L}_{n}\left(\widehat{\beta}^{\mathcal{O}}\right)-\nabla \mathcal{L}_{n}\left(\beta^{*}\right)\right)_{S}+\left(\nabla \mathcal{L}_{n}\left(\beta^{*}\right)\right)_{S}=0
$$

or

$$
\widehat{Q}_{S S}^{\mathcal{O}}\left(\widehat{\beta}^{\mathcal{O}}-\beta^{*}\right)_{S}+\left(\nabla \mathcal{L}_{n}\left(\beta^{*}\right)\right)_{S}=0
$$

where

$$
\widehat{Q}^{\mathcal{O}}:=\int_{0}^{1} \nabla^{2} \mathcal{L}_{n}\left(\beta^{*}+t\left(\widehat{\beta}^{\mathcal{O}}-\beta^{*}\right)\right) d t=\left(\int_{0}^{1} \ell^{\prime \prime}\left(x_{i}^{T}\left(\beta^{*}+t\left(\widehat{\beta}^{\mathcal{O}}-\beta^{*}\right)\right)-y_{i}\right) d t\right) \cdot x_{i} x_{i}^{T} .
$$

This implies that

$$
\left\|\widehat{\beta}_{S}^{\mathcal{O}}-\beta_{S}^{*}\right\|_{\infty}=\left\|\left(\widehat{Q}^{\mathcal{O}}\right)_{S S}^{-1}\left(\nabla \mathcal{L}_{n}\left(\beta^{*}\right)\right)_{S}\right\|_{\infty}
$$


Let $\mathbb{B}_{2}^{S}(1):=\left\{u:\|u\|_{2} \leq 1\right.$, and $\left.\operatorname{supp}(u) \subseteq S\right\}$. For $v, w \in \mathbb{B}_{2}^{S}(1)$, consider the quantity

$$
\begin{aligned}
\mid v^{T}\left\{\widehat{Q}_{S S}^{\mathcal{O}}-\right. & \left.\left(\nabla^{2} \mathcal{L}_{n}\left(\beta^{*}\right)\right)_{S S}\right\} w \mid \\
& =\left|\frac{1}{n} \sum_{i=1}^{n}\left\{\int_{0}^{1}\left(\ell^{\prime \prime}\left(x_{i}^{T}\left(\beta^{*}+t\left(\widehat{\beta}^{\mathcal{O}}-\beta^{*}\right)\right)-y_{i}\right)-\ell^{\prime \prime}\left(x_{i}^{T} \beta^{*}-y_{i}\right)\right) d t\right\}\left(x_{i}^{T} v\right)\left(x_{i}^{T} w\right)\right| \\
& \leq \kappa_{3} \cdot \frac{1}{n} \sum_{i=1}^{n} \int_{0}^{1}\left(t \cdot\left|x_{i}^{T}\left(\widehat{\beta}^{\mathcal{O}}-\beta^{*}\right)\right| d t\right) \cdot\left|x_{i}^{T} v\right| \cdot\left|x_{i}^{T} w\right| \\
& =\frac{\kappa_{3}}{2} \cdot \frac{1}{n} \sum_{i=1}^{n}\left|x_{i}^{T}\left(\widehat{\beta}^{\mathcal{O}}-\beta^{*}\right)\right| \cdot\left|x_{i}^{T} v\right| \cdot\left|x_{i}^{T} w\right| \\
& \leq \frac{\kappa_{3}}{2}\left\|\widehat{\beta}^{\mathcal{O}}-\beta^{*}\right\|_{2} \cdot \sup _{\|u\|_{2}=1, \operatorname{supp}(u) \subseteq S}\left\{\frac{1}{n} \sum_{i=1}^{n}\left|x_{i}^{T} u\right| \cdot\left|x_{i}^{T} v\right| \cdot\left|x_{i}^{T} w\right|\right\},
\end{aligned}
$$

and denote $f(u, v, w):=\frac{1}{n} \sum_{i=1}^{n}\left|x_{i}^{T} u\right| \cdot\left|x_{i}^{T} v\right| \cdot\left|x_{i}^{T} w\right|$. Then

$$
\left\|\widehat{Q}_{S S}^{\mathcal{O}}-\left(\nabla^{2} \mathcal{L}_{n}\left(\beta^{*}\right)\right)_{S S}\right\|_{2} \leq \frac{\kappa_{3}}{2}\left\|\widehat{\beta}^{\mathcal{O}}-\beta^{*}\right\|_{2} \cdot \sup _{u, v, w \in \mathbb{B}_{2}^{S}(1)} f(u, v, w) .
$$

We now use a covering argument. Let $\mathcal{M}$ denote a $\frac{1}{4}$-cover of $\mathbb{B}_{2}(1)$. By standard results on metric entropy, we may choose $\mathcal{M}$ such that $|\mathcal{M}| \leq c^{k}$. For all triples $u, v, w \in \mathbb{B}_{2}^{S}(1)$, we may find $u^{\prime}, v^{\prime}, w^{\prime} \in \mathcal{M}$ such that

$$
\left\|u-u^{\prime}\right\|_{2},\left\|v-v^{\prime}\right\|_{2},\left\|w-w^{\prime}\right\|_{2} \leq \frac{1}{4} .
$$

Furthermore,

$$
\begin{aligned}
\left|f(u, v, w)-f\left(u^{\prime}, v^{\prime}, w^{\prime}\right)\right| & \leq\left|f(u, v, w)-f\left(u^{\prime}, v, w\right)\right| \\
& +\left|f\left(u^{\prime}, v, w\right)-f\left(u^{\prime}, v^{\prime}, w\right)\right|+\left|f\left(u^{\prime}, v^{\prime}, w\right)-f\left(u^{\prime}, v^{\prime}, w^{\prime}\right)\right|
\end{aligned}
$$


Note that

$$
\begin{aligned}
\left|f(u, v, w)-f\left(u^{\prime}, v, w\right)\right| & =\left|\frac{1}{n} \sum_{i=1}^{n}\left(\left|x_{i}^{T} u\right|-\left|x_{i}^{T} u^{\prime}\right|\right) \cdot\right| x_{i}^{T} v|\cdot| x_{i}^{T} w|| \\
& \leq \frac{1}{n} \sum_{i=1}^{n}|| x_{i}^{T} u|-| x_{i}^{T} u^{\prime}|| \cdot\left|x_{i}^{T} v\right| \cdot\left|x_{i}^{T} w\right| \\
& \leq \frac{1}{n} \sum_{i=1}^{n}\left|x_{i}^{T}\left(u-u^{\prime}\right)\right| \cdot\left|x_{i}^{T} v\right| \cdot\left|x_{i}^{T} w\right| \\
& \leq\left\|u-u^{\prime}\right\|_{2} \cdot \sup _{u, v, w \in \mathbb{B}_{2}^{S}(1)} f(u, v, w) \\
& \leq \frac{1}{4} \cdot \sup _{u, v, w \in \mathbb{B}_{2}^{S}(1)} f(u, v, w)
\end{aligned}
$$

and we may bound the other two terms in the expansion (50) analogously. Hence,

$$
\sup _{u, v, w \in \mathbb{B}_{2}^{S}(1)} f(u, v, w) \leq \max _{u^{\prime}, v^{\prime}, w^{\prime} \in \mathcal{M}} f\left(u^{\prime}, v^{\prime}, w^{\prime}\right)+\frac{3}{4} \cdot \sup _{u, v, w \in \mathbb{B}_{2}^{S}(1)} f(u, v, w),
$$

implying that

$$
\sup _{u, v, w \in \mathbb{B}_{2}^{S}(1)} f(u, v, w) \leq 4 \cdot \max _{u, v, w \in \mathcal{M}} f(u, v, w),
$$

and it remains to bound the right-hand side of inequality (51). For a fixed triple $u, v, w \in$ $\mathcal{M}$, we apply the arithmetic mean-geometric mean inequality, to obtain

$$
\left|x_{i}^{T} u\right| \cdot\left|x_{i}^{T} v\right| \cdot\left|x_{i}^{T} w\right| \leq \frac{1}{3}\left(\left|x_{i}^{T} u\right|^{3}+\left|x_{i}^{T} v\right|^{3}+\left|x_{i}^{T} w\right|^{3}\right) .
$$

Then

$$
f(u, v, w) \leq \frac{1}{3}\left(\frac{1}{n} \sum_{i=1}^{n}\left|x_{i}^{T} u\right|^{3}+\frac{1}{n} \sum_{i=1}^{n}\left|x_{i}^{T} v\right|^{3}+\frac{1}{n} \sum_{i=1}^{n}\left|x_{i}^{T} w\right|^{3}\right)
$$

Note that

$$
\mathbb{E}[f(u, v, w)]=\frac{1}{3}\left(\mathbb{E}\left[\left|x_{i}^{T} u\right|^{3}\right]+\mathbb{E}\left[\left|x_{i}^{T} v\right|^{3}\right]+\mathbb{E}\left[\left|x_{i}^{T} w\right|^{3}\right]\right) \leq c \sigma_{x}^{3},
$$

using the sub-Gaussian assumption on the $x_{i}$ 's. Finally, we invoke a concentration bound on $f(u, v, w)$. We use a result from Adamczak and Wolff (2014). Theorem 1.4 of that paper gives a concentration result for i.i.d. averages of polynomials of sub-Gaussian variables, implying in particular that

$$
\mathbb{P}(|f(u, v, w)-\mathbb{E}[f(u, v, w)]| \geq t) \leq c_{1} \exp \left(\min \left\{\frac{n t^{2}}{\sigma_{x}^{6}}, \frac{(n t)^{2 / 3}}{\sigma_{x}^{2}}\right\}\right), \quad \forall t>0 .
$$


Setting $t=c \sigma_{x}^{3} \sqrt{\frac{k}{n}}$ and taking a union bound over all $u, v, w \in \mathcal{M}$, we then conclude from inequality (51) that

$$
\sup _{u, v, w \in \mathbb{B}_{2}^{S}(1)} f(u, v, w) \leq c \sigma_{x}^{3} \sqrt{\frac{k}{n}},
$$

with probability at least $1-c_{1}^{\prime} \exp \left(-c_{2}^{\prime} k\right)$. Plugging back into inequality (49) then gives

$$
\left\|\widehat{Q}_{S S}^{\mathcal{O}}-\left(\nabla^{2} \mathcal{L}_{n}\left(\beta^{*}\right)\right)_{S S}\right\|_{2} \leq c^{\prime} \kappa_{3} \sigma_{x}^{3}\left\|\widehat{\beta}^{\mathcal{O}}-\beta^{*}\right\|_{2} \cdot \sqrt{\frac{k}{n}} \leq c^{\prime} \kappa_{3} \sigma_{x}^{3} r \sqrt{\frac{k}{n}} .
$$

We further note that

$$
v^{T}\left\{\left(\nabla^{2} \mathcal{L}_{n}\left(\beta^{*}\right)\right)_{S S}\right\} w=\frac{1}{n} \sum_{i=1}^{n} \ell^{\prime \prime}\left(x_{i}^{T} \beta^{*}-y_{i}\right) \cdot\left(x_{i}^{T} v\right) \cdot\left(x_{i}^{T} w\right),
$$

which is an i.i.d. average of products of sub-Gaussians (since $\ell^{\prime \prime}$ is bounded), so an even easier covering argument establishes concentration to $v^{T}\left\{\left(\nabla^{2} \mathcal{L}\left(\beta^{*}\right)\right)_{S S}\right\} w$. Hence,

$$
\left\|\widehat{Q}_{S S}^{\mathcal{O}}-\left(\nabla^{2} \mathcal{L}\left(\beta^{*}\right)\right)_{S S}\right\|_{2} \leq c^{\prime \prime} \sqrt{\frac{k}{n}}
$$

Combining inequalities (52) and (53) gives

$$
\left\|\widehat{Q}_{S S}^{\mathcal{O}}-\left(\nabla^{2} \mathcal{L}\left(\beta^{*}\right)\right)_{S S}\right\|_{2} \leq c^{\prime \prime \prime} \sqrt{\frac{k}{n}}
$$

Then by a simple matrix inversion relation (cf. Lemma 12 in Loh and Wainwright (2014)), we have

$$
\left\|\left(\widehat{Q}_{S S}^{\mathcal{O}}\right)^{-1}-\left(\nabla^{2} \mathcal{L}\left(\beta^{*}\right)\right)_{S S}^{-1}\right\|_{2} \leq c^{\prime \prime \prime} \sqrt{\frac{k}{n}}
$$

as well. Returning to equation (48), we see that

$$
\begin{aligned}
\| \widehat{\beta}_{S}^{\mathcal{O}} & -\beta_{S}^{*} \|_{\infty} \\
& \leq\left\|\left\{\left(\widehat{Q}_{S S}^{\mathcal{O}}\right)^{-1}-\left(\nabla^{2} \mathcal{L}\left(\beta^{*}\right)\right)_{S S}^{-1}\right\}\left(\nabla \mathcal{L}_{n}\left(\beta^{*}\right)\right)_{S}\right\|_{\infty}+\left\|\left(\nabla^{2} \mathcal{L}\left(\beta^{*}\right)\right)_{S S}^{-1}\left(\nabla \mathcal{L}_{n}\left(\beta^{*}\right)\right)_{S}\right\|_{\infty} \\
& \leq c^{\prime \prime \prime} \sqrt{\frac{k}{n}} \cdot \sqrt{k}\left\|\left(\nabla \mathcal{L}_{n}\left(\beta^{*}\right)\right)_{S}\right\|_{\infty}+\left\|\left(\nabla^{2} \mathcal{L}\left(\beta^{*}\right)\right)_{S S}^{-1}\left(\nabla \mathcal{L}_{n}\left(\beta^{*}\right)\right)_{S}\right\|_{\infty} \\
& \leq C \sqrt{\frac{\log k}{n}}
\end{aligned}
$$

assuming $n \geq k^{2}$. This is the desired result. 
In particular, Lemma 3 implies when $\beta_{\min }^{*} \geq C \sqrt{\frac{\log k}{n}}+\gamma \lambda$, we have

$$
\nabla q_{\lambda}\left(\widehat{\beta}_{S}\right)=\lambda \operatorname{sign}\left(\widehat{\beta}_{S}\right)=\lambda \widehat{z}_{S}
$$

Furthermore, the selection property implies $\nabla q_{\lambda}\left(\widehat{\beta}_{S^{c}}\right)=0$. Plugging these results into equation (45) and performing some algebra, we conclude that

$$
\widehat{z}_{S^{c}}=\frac{1}{\lambda}\left\{\widehat{Q}_{S^{c} S}\left(\widehat{Q}_{S S}\right)^{-1}\left(\nabla \mathcal{L}_{n}\left(\beta^{*}\right)\right)_{S}-\left(\nabla \mathcal{L}_{n}\left(\beta^{*}\right)\right)_{S^{c}}\right\}
$$

so

$$
\left\|\widehat{z}_{S^{c}}\right\|_{\infty} \leq \frac{1}{\lambda}\left\|\widehat{Q}_{S^{c} S}\left(\widehat{Q}_{S S}\right)^{-1}\left(\nabla \mathcal{L}_{n}\left(\beta^{*}\right)\right)_{S}\right\|_{\infty}+\frac{1}{\lambda}\left\|\left(\nabla \mathcal{L}_{n}\left(\beta^{*}\right)\right)_{S^{c}}\right\|_{\infty}
$$

We now use similar arguments to those employed in the proof of Lemma 3 to control the terms in inequality $(55)$. Note that $\left\|\nabla \mathcal{L}_{n}\left(\beta^{*}\right)\right\|_{\infty} \leq c \sqrt{\frac{\log p}{n}}$ by assumption, so we can focus on the first term. We have

$$
\begin{aligned}
\left|v^{T}\left(\widehat{Q}-\nabla^{2} \mathcal{L}_{n}\left(\beta^{*}\right)\right) w\right| \\
\quad=\left|\frac{1}{n} \sum_{i=1}^{n} \int_{0}^{1}\left(\ell^{\prime \prime}\left(x_{i}^{T}\left(\widehat{\beta}+t\left(\widehat{\beta}-\beta^{*}\right)\right)-y_{i}\right)-\ell^{\prime \prime}\left(x_{i}^{T} \beta^{*}-y_{i}\right)\right) d t \cdot\left(x_{i}^{T} v\right)\left(x_{i}^{T} w\right)\right| \\
\leq \kappa_{3} \cdot \frac{1}{n} \sum_{i=1}^{n} \int_{0}^{1}\left(t \cdot\left|x_{i}^{T}\left(\widehat{\beta}-\beta^{*}\right)\right|\right) d t \cdot\left|x_{i}^{T} v\right| \cdot\left|x_{i}^{T} w\right| \\
\leq \kappa_{3}\left\|\widehat{\beta}-\beta^{*}\right\|_{2} \cdot \sup _{u \in \mathbb{B}_{2}^{S}(1)}\left\{\frac{1}{n} \sum_{i=1}^{n}\left|x_{i}^{T} u\right| \cdot\left|x_{i}^{T} v\right| \cdot\left|x_{i}^{T} w\right|\right\} \\
\quad \leq \kappa_{3} r \cdot \sup _{u \in \mathbb{B}_{2}^{S}(1)}\left\{\frac{1}{n} \sum_{i=1}^{n}\left|x_{i}^{T} u\right| \cdot\left|x_{i}^{T} v\right| \cdot\left|x_{i}^{T} w\right|\right\} .
\end{aligned}
$$

By essentially the same bounding and covering argument as before, we conclude that

$$
\left\|\widehat{Q}_{S S}-\left(\nabla^{2} \mathcal{L}\left(\beta^{*}\right)\right)_{S S}\right\|_{2} \leq c \sqrt{\frac{k}{n}},
$$

and

$$
\left\|\left(\widehat{Q}_{S S}\right)^{-1}-\left(\nabla^{2} \mathcal{L}\left(\beta^{*}\right)\right)_{S S}^{-1}\right\|_{2} \leq c^{\prime} \sqrt{\frac{k}{n}},
$$

with probability at least $1-c_{1} \exp \left(-c_{2} k\right)$. Furthermore, we may show that

$$
\max _{j \in S^{c}}\left\|e_{j}^{T}\left\{\widehat{Q}_{S^{c} S}-\left(\nabla^{2} \mathcal{L}\left(\beta^{*}\right)\right)_{S^{c} S}\right\}\right\|_{2} \leq c^{\prime \prime \prime} \sqrt{\frac{k+\log p}{n}},
$$


with probability at least $1-c_{1}^{\prime} \exp \left(-c_{2}^{\prime} \min \{k, \log p\}\right)$ by a similar argument, this time taking a union bound over $j \in S^{c}$ rather than all unit vectors for one of the coordinates in the covering. Defining

$$
\delta_{1}:=\widehat{Q}_{S^{c} S}-\left(\nabla^{2} \mathcal{L}\left(\beta^{*}\right)\right)_{S^{c} S}, \quad \text { and } \quad \delta_{2}:=\left(\widehat{Q}_{S S}\right)^{-1}-\left(\nabla^{2} \mathcal{L}\left(\beta^{*}\right)\right)_{S S}^{-1},
$$

we may conclude that

$$
\begin{aligned}
& \left\|\widehat{Q}_{S^{c} S}\left(\widehat{Q}_{S S}\right)^{-1}\left(\nabla \mathcal{L}_{n}\left(\beta^{*}\right)\right)_{S}\right\|_{\infty} \leq\left\|\delta_{1} \delta_{2}\left(\nabla \mathcal{L}_{n}\left(\beta^{*}\right)\right)_{S}\right\|_{\infty}+\left\|\delta_{1}\left(\nabla^{2} \mathcal{L}\left(\beta^{*}\right)\right)_{S S}^{-1}\left(\nabla \mathcal{L}_{n}\left(\beta^{*}\right)\right)_{S}\right\|_{\infty} \\
& \quad+\left\|\left(\nabla^{2} \mathcal{L}\left(\beta^{*}\right)\right)_{S^{c} S} \delta_{2}\left(\nabla \mathcal{L}_{n}\left(\beta^{*}\right)\right)_{S}\right\|_{\infty} \\
& \leq \max _{j \in S^{c}}\left\|e_{j}^{T} \delta_{1}\right\|_{2} \cdot\left\|\delta_{2}\right\|_{2} \cdot \sqrt{k}\left\|\nabla \mathcal{L}_{n}\left(\beta^{*}\right)\right\|_{\infty}+\max _{j \in S^{c}}\left\|e_{j}^{T} \delta_{1}\right\|_{2} \cdot \sqrt{k}\left\|\left(\nabla^{2} \mathcal{L}\left(\beta^{*}\right)\right)_{S S}^{-1}\left(\nabla \mathcal{L}_{n}\left(\beta^{*}\right)\right)_{S}\right\|_{\infty} \\
& \quad+\left\|\left(\nabla^{2} \mathcal{L}\left(\beta^{*}\right)\right)_{S^{c} S}\right\|_{2} \cdot\left\|\delta_{2}\right\|_{2} \cdot \sqrt{k}\left\|\nabla \mathcal{L}_{n}\left(\beta^{*}\right)\right\|_{\infty} \\
& \leq C \\
& \quad \sqrt{\frac{\log p}{n}}
\end{aligned}
$$

with probability at least $1-c_{1}^{\prime} \exp \left(-c_{2}^{\prime} \min \{k, \log p\}\right)$, assuming the scaling $n \geq \max \left\{k^{2}, k \log p\right\}$ and using the inqualities (56) and (57) above. In particular, for $\lambda \geq C^{\prime} \sqrt{\frac{\log p}{n}}$, we conclude at last that the strict dual feasibility condition $\left\|\widehat{z}_{S^{c}}\right\|_{\infty}<1$ holds, completing step (ii) of the PDW construction.

Step (iii). Finally, we establish that $\widehat{\beta}=\left(\widehat{\beta}_{S}, 0_{S^{c}}\right)$ is a local minimum of the full program (2) and in fact, all stationary points of the program must take this form. A classical result by Fletcher and Watson (1980) gives sufficient conditions for a point to be a local minimum of a norm-regularized program. Rather than repeating the details here, we refer the reader to the argument provided in the proof of Theorem 1 in Loh and Wainwright (2014), which may be applied verbatim to establish that $\widehat{\beta}$ is a local minimum. Now suppose $\widetilde{\beta}$ is a stationary point of the program (2) satisfying $\left\|\widetilde{\beta}-\beta^{*}\right\|_{2} \leq r$. By Assumption 2 applied to the pair $(\widetilde{\beta}, \widehat{\beta})$, we have

$$
\alpha\|\widetilde{\beta}-\widehat{\beta}\|_{2}^{2}-\tau \frac{\log p}{n}\|\widetilde{\beta}-\widehat{\beta}\|_{1}^{2} \leq\left\langle\nabla \mathcal{L}_{n}(\widetilde{\beta})-\nabla \mathcal{L}_{n}(\widehat{\beta}), \widetilde{\beta}-\widehat{\beta}\right\rangle .
$$

By the convexity of $\frac{\mu}{2}\|\beta\|_{2}^{2}-q_{\lambda}(\beta)$, we also have

$$
\left\langle\nabla q_{\lambda}(\widetilde{\beta})-\nabla q_{\lambda}(\widehat{\beta}), \widetilde{\beta}-\widehat{\beta}\right\rangle \leq \mu\|\widetilde{\beta}-\widehat{\beta}\|_{2}^{2}
$$

Finally, the first-order optimality condition applied to $\widetilde{\beta}$ gives

$$
0 \leq\left\langle\nabla \mathcal{L}_{n}(\widetilde{\beta})-\nabla q_{\lambda}(\widetilde{\beta}), \widehat{\beta}-\widetilde{\beta}\right\rangle+\lambda \cdot\langle\widetilde{z}, \widehat{\beta}-\widetilde{\beta}\rangle,
$$


where $\widetilde{z} \in \partial\|\widetilde{\beta}\|_{1}$. Summing the inequalities (58), (59), and (60), we obtain

$$
(\alpha-\mu)\|\widetilde{\beta}-\widehat{\beta}\|_{2}^{2}-\tau \frac{\log p}{n}\|\widetilde{\beta}-\widehat{\beta}\|_{1}^{2} \leq\left\langle\nabla q_{\lambda}(\widehat{\beta})-\nabla \mathcal{L}_{n}(\widehat{\beta}), \widetilde{\beta}-\widehat{\beta}\right\rangle+\lambda \cdot\langle\widetilde{z}, \widehat{\beta}-\widetilde{\beta}\rangle .
$$

Recall that since $\widehat{\beta}$ is an interior point, we have the zero-subgradient condition

$$
\nabla \mathcal{L}_{n}(\widehat{\beta})-\nabla q_{\lambda}(\widehat{\beta})+\lambda \widehat{z}=0 .
$$

Combining this with inequality (61), we obtain

$$
\begin{aligned}
(\alpha-\mu)\|\widetilde{\beta}-\widehat{\beta}\|_{2}^{2}-\tau \frac{\log p}{n}\|\widetilde{\beta}-\widehat{\beta}\|_{1}^{2} & \leq \lambda \cdot\langle\widehat{z}, \widetilde{\beta}-\widehat{\beta}\rangle+\lambda \cdot\langle\widetilde{z}, \widehat{\beta}-\widetilde{\beta}\rangle \\
& =\lambda \cdot\langle\widehat{z}, \widetilde{\beta}\rangle-\lambda\|\widehat{\beta}\|_{1}+\lambda \cdot\langle\widetilde{z}, \widehat{\beta}\rangle-\lambda\|\widetilde{\beta}\|_{1} \\
& \leq \lambda \cdot\langle\widehat{z}, \widetilde{\beta}\rangle-\lambda\|\widetilde{\beta}\|_{1} .
\end{aligned}
$$

We now show the following lemma:

Lemma 4. Suppose $\delta>0$ is such that $\left\|\widehat{z}_{S^{c}}\right\|_{\infty} \leq 1-\delta$. Then for $\lambda \geq \frac{4 R \tau}{\log p} \delta n$, we have

$$
\|\widetilde{\beta}-\widehat{\beta}\|_{1} \leq\left(\frac{4}{\delta}+2\right) \sqrt{k}\|\widetilde{\beta}-\widehat{\beta}\|_{2}
$$

Proof. This is identical to the proof of Lemma 7 in Loh and Wainwright (2014).

Using Lemma 4 to bound the left-hand side of inequality (62), we then obtain

$$
\left(\alpha-\mu-\tau \frac{k \log p}{n}\left(\frac{4}{\delta}+2\right)^{2}\right)\|\widetilde{\beta}-\widehat{\beta}\|_{2}^{2} \leq \lambda \cdot\langle\widehat{z}, \widetilde{\beta}\rangle-\lambda\|\widetilde{\beta}\|_{1},
$$

so if $n \geq \frac{2 \tau}{\alpha-\mu}\left(\frac{4}{\delta}+2\right)^{2} k \log p$, this implies

$$
0 \leq \lambda \cdot\langle\widehat{z}, \widetilde{\beta}\rangle-\lambda\|\widetilde{\beta}\|_{1}
$$

At the same time, Hölder's inequality gives

$$
\lambda \cdot\langle\widehat{z}, \widetilde{\beta}\rangle-\lambda\|\widetilde{\beta}\|_{1} \leq \lambda \cdot\|\widehat{z}\|_{\infty}\|\widetilde{\beta}\|_{1}-\lambda\|\widetilde{\beta}\|_{1} \leq \lambda\|\widetilde{\beta}\|_{1}-\lambda\|\widetilde{\beta}\|_{1}=0 .
$$

Hence, we must have $\langle\widehat{z}, \widetilde{\beta}\rangle=\|\widetilde{\beta}\|_{1}$. Since $\left\|\widehat{z}_{S^{c}}\right\|_{\infty}<1$ by assumption, this means that $\operatorname{supp}(\widetilde{\beta}) \subseteq S$, as wanted. 
C.3. Proof of Theorem 3. We derive the following variants of Lemmas 1 and 2 in Loh and Wainwright (2015); the remainder of the argument is exactly the same as in that paper, so we do not repeat it here. The reason why we need to revise the two lemmas is that the proofs in Loh and Wainwright (2015) require the statement of the RSC condition in that paper, which also provides control on the behavior of $\mathcal{L}_{n}$ outside the local region.

LEMMA 5. Under the conditions of the theorem, we have

$$
\left\|\beta^{t}-\widehat{\beta}\right\|_{2} \leq \frac{r}{2}, \quad \forall t \geq 0
$$

Proof. We induct on the iteration number $t$. Note that the base case, $t=0$, holds by assumption. Suppose $t \geq 0$ is such that $\left\|\beta^{t}-\widehat{\beta}\right\|_{2} \leq \frac{r}{2}$; we will show that $\left\|\beta^{t+1}-\widehat{\beta}\right\|_{2} \leq \frac{r}{2}$, as well.

By the RSC' condition (22), we have

$$
\alpha^{\prime}\left\|\beta^{t}-\widehat{\beta}\right\|_{2}^{2}-\tau^{\prime} \frac{\log p}{n}\left\|\beta^{t}-\widehat{\beta}\right\|_{1}^{2} \leq \mathcal{L}_{n}(\widehat{\beta})-\mathcal{L}_{n}\left(\beta^{t}\right)-\left\langle\nabla \mathcal{L}_{n}\left(\beta^{t}\right), \widehat{\beta}-\beta^{t}\right\rangle .
$$

Furthermore, since $\frac{\mu}{2}\|\beta\|_{2}^{2}-q_{\lambda}(\beta)$ is convex by the $\mu$-amenability of $\rho_{\lambda}$, combining inequality (69) with $\left(\beta_{1}, \beta_{2}\right)=\left(\beta^{t}, \widehat{\beta}\right)$ and inequality (63) and the inequality

$$
\left\|\beta^{t+1}\right\|_{1}+\left\langle\operatorname{sign}\left(\beta^{t+1}\right), \widehat{\beta}-\beta^{t+1}\right\rangle \leq\|\widehat{\beta}\|_{1}
$$

implies that

$$
\begin{aligned}
\mathcal{L}_{n}\left(\beta^{t}\right)+\left\langle\nabla \mathcal{L}_{n}\left(\beta^{t}\right)\right. & \left.-\nabla q_{\lambda}\left(\beta^{t}\right), \widehat{\beta}-\beta^{t}\right\rangle-q_{\lambda}\left(\beta^{t}\right)+\lambda\left\|\beta^{t+1}\right\|_{1}+\lambda\left\langle\operatorname{sign}\left(\beta^{t+1}\right), \widehat{\beta}-\beta^{t+1}\right\rangle \\
+ & \left(\alpha^{\prime}-\frac{\mu}{2}\right)\left\|\beta^{t}-\widehat{\beta}\right\|_{2}^{2}-\tau^{\prime} \frac{\log p}{n}\left\|\beta^{t}-\widehat{\beta}\right\|_{1}^{2} \leq \mathcal{L}_{n}(\widehat{\beta})-q_{\lambda}(\widehat{\beta})+\lambda\|\widehat{\beta}\|_{1},
\end{aligned}
$$

$\mathrm{SO}$

$$
\begin{aligned}
\overline{\mathcal{L}}_{n}\left(\beta^{t}\right)+\left\langle\nabla \overline{\mathcal{L}}_{n}\left(\beta^{t}\right), \widehat{\beta}-\beta^{t}\right\rangle+\lambda\left\|\beta^{t+1}\right\|_{1}+\lambda\left\langle\operatorname{sign}\left(\beta^{t+1}\right), \widehat{\beta}-\beta^{t+1}\right\rangle & -\tau^{\prime} \frac{\log p}{n}\left\|\beta^{t}-\widehat{\beta}\right\|_{1}^{2} \\
& \leq \overline{\mathcal{L}}_{n}(\widehat{\beta})+\lambda\|\widehat{\beta}\|_{1} .
\end{aligned}
$$

By the RSM condition (23), we have

$$
\mathcal{L}_{n}\left(\beta^{t+1}\right)-\mathcal{L}_{n}\left(\beta^{t}\right)-\left\langle\nabla \mathcal{L}_{n}\left(\beta^{t}\right), \beta^{t+1}-\beta^{t}\right\rangle \leq \alpha^{\prime \prime}\left\|\beta^{t+1}-\beta^{t}\right\|_{2}^{2}+\tau^{\prime \prime} \frac{\log p}{n}\left\|\beta^{t+1}-\beta^{t}\right\|_{1}^{2},
$$

and combined with the convexity of $q_{\lambda}$, we have

$$
\overline{\mathcal{L}}_{n}\left(\beta^{t+1}\right)-\overline{\mathcal{L}}_{n}\left(\beta^{t}\right)-\left\langle\nabla \overline{\mathcal{L}}_{n}\left(\beta^{t}\right), \beta^{t+1}-\beta^{t}\right\rangle \leq \alpha^{\prime \prime}\left\|\beta^{t+1}-\beta^{t}\right\|_{2}^{2}+\tau^{\prime \prime} \frac{\log p}{n}\left\|\beta^{t+1}-\beta^{t}\right\|_{1}^{2}
$$


Combining inequalities (64) and (65) then gives

$$
\begin{aligned}
& (66) \quad\left(\overline{\mathcal{L}}_{n}\left(\beta^{t+1}\right)+\lambda\left\|\beta^{t+1}\right\|_{1}\right)-\left(\overline{\mathcal{L}}_{n}(\widehat{\beta})+\lambda\|\widehat{\beta}\|_{1}\right) \\
& \leq\left\langle\nabla \overline{\mathcal{L}}_{n}\left(\beta^{t}\right), \beta^{t+1}-\widehat{\beta}\right\rangle+\lambda\left\langle\operatorname{sign}\left(\beta^{t+1}\right), \beta^{t+1}-\widehat{\beta}\right\rangle+\alpha^{\prime \prime}\left\|\beta^{t+1}-\beta^{t}\right\|_{2}^{2}+4 R^{2}\left(\tau^{\prime}+\tau^{\prime \prime}\right) \frac{\log p}{n},
\end{aligned}
$$

using the fact that $\left\|\beta^{t+1}-\beta^{t}\right\|_{1},\left\|\beta^{t}-\widehat{\beta}\right\|_{1} \leq 2 R$, by feasibility of each point. Note that the left-hand side of inequality (66) is lower-bounded by 0 , since $\widehat{\beta}$ is a global optimum. Finally, note that from the first-order optimality condition on equation (20), we have

$$
\left\langle\nabla \overline{\mathcal{L}}_{n}\left(\beta^{t}\right)+\eta\left(\beta^{t+1}-\beta^{t}\right)+\lambda \operatorname{sign}\left(\beta^{t+1}\right), \beta^{t+1}-\widehat{\beta}\right\rangle \leq 0 .
$$

Combining inequality (67) with inequality (66) then gives

$$
\begin{aligned}
0 & \leq\left(\overline{\mathcal{L}}_{n}\left(\beta^{t+1}\right)+\lambda\left\|\beta^{t+1}\right\|_{1}\right)-\left(\overline{\mathcal{L}}_{n}(\widehat{\beta})+\lambda\|\widehat{\beta}\|_{1}\right) \\
& \leq \alpha^{\prime \prime}\left\|\beta^{t+1}-\beta^{t}\right\|_{2}^{2}+\left(\tau^{\prime}+\tau^{\prime \prime}\right) \frac{4 R^{2} \log p}{n}-\eta\left\langle\beta^{t+1}-\beta^{t}, \beta^{t+1}-\widehat{\beta}\right\rangle \\
& =\left(\alpha^{\prime \prime}-\frac{\eta}{2}\right)\left\|\beta^{t+1}-\beta^{t}\right\|_{2}^{2}-\frac{\eta}{2}\left\|\beta^{t+1}-\widehat{\beta}\right\|_{2}^{2}+\frac{\eta}{2}\left\|\beta^{t}-\widehat{\beta}\right\|_{2}^{2}+\left(\tau^{\prime}+\tau^{\prime \prime}\right) \frac{4 R^{2} \log p}{n} \\
& \leq \frac{\eta}{2}\left\|\beta^{t}-\widehat{\beta}\right\|_{2}^{2}-\frac{\eta}{2}\left\|\beta^{t+1}-\widehat{\beta}\right\|_{2}^{2}+\left(\tau^{\prime}+\tau^{\prime \prime}\right) \frac{4 R^{2} \log p}{n}
\end{aligned}
$$

using the assumption that $\eta \geq 2 \alpha^{\prime \prime}$. Hence,

$$
\left\|\beta^{t+1}-\widehat{\beta}\right\|_{2}^{2} \leq\left\|\beta^{t}-\widehat{\beta}\right\|_{2}^{2}+\frac{8\left(\tau^{\prime}+\tau^{\prime \prime}\right)}{\eta} \cdot \frac{R^{2} \log p}{n} .
$$

Using the inductive hypothesis and the assumption that $n \geq \frac{32\left(\tau^{\prime}+\tau^{\prime \prime}\right) R^{2}}{\eta r^{2}} \log p$, we then have

$$
\left\|\beta^{t+1}-\widehat{\beta}\right\|_{2}^{2} \leq \frac{r^{2}}{4}+\frac{r^{2}}{4} \leq r^{2} .
$$

In particular, we may apply the RSC' condition $(22)$ to the pair $\left(\beta^{t+1}, \widehat{\beta}\right)$ to obtain

$$
\alpha^{\prime}\left\|\beta^{t+1}-\widehat{\beta}\right\|_{2}^{2}-\tau^{\prime} \frac{\log p}{n}\left\|\beta^{t+1}-\widehat{\beta}\right\|_{1}^{2} \leq \mathcal{L}_{n}\left(\beta^{t+1}\right)-\mathcal{L}_{n}(\widehat{\beta})-\left\langle\nabla \mathcal{L}_{n}(\widehat{\beta}), \beta^{t+1}-\widehat{\beta}\right\rangle .
$$

By the convexity of $\frac{\mu}{2}\|\beta\|_{2}^{2}-q_{\lambda}(\beta)$, we have

$$
\left\langle\nabla q_{\lambda}(\widehat{\beta}), \beta^{t+1}-\widehat{\beta}\right\rangle \geq q_{\lambda}\left(\beta^{t+1}\right)-q_{\lambda}(\widehat{\beta})-\frac{\mu}{2}\left\|\widehat{\beta}-\beta^{t+1}\right\|_{2}^{2} .
$$

Together with the inequality

$$
\|\widehat{\beta}\|_{1}+\left\langle\operatorname{sign}(\widehat{\beta}), \beta^{t+1}-\widehat{\beta}\right\rangle \leq\left\|\beta^{t+1}\right\|_{1},
$$


we then have

$$
\begin{aligned}
& \left(\alpha^{\prime}-\frac{\mu}{2}\right)\left\|\beta^{t+1}-\widehat{\beta}\right\|_{2}^{2}-\tau^{\prime} \frac{\log p}{n}\left\|\beta^{t+1}-\widehat{\beta}\right\|_{1}^{2} \\
& \leq\left(\overline{\mathcal{L}}_{n}\left(\beta^{t+1}\right)+\lambda\left\|\beta^{t+1}\right\|_{1}\right)-\left(\overline{\mathcal{L}}_{n}(\widehat{\beta})+\lambda\|\widehat{\beta}\|_{1}\right)-\left\langle\nabla \overline{\mathcal{L}}_{n}(\widehat{\beta})+\lambda \operatorname{sign}(\widehat{\beta}), \beta^{t+1}-\widehat{\beta}\right\rangle .
\end{aligned}
$$

Finally, the first-order optimality condition on $\widehat{\beta}$ gives

$$
\left\langle\nabla \overline{\mathcal{L}}_{n}(\widehat{\beta})+\lambda \operatorname{sign}(\widehat{\beta}), \beta^{t+1}-\widehat{\beta}\right\rangle \geq 0 .
$$

Combined with inequality (70), we conclude that

$$
\left(\alpha^{\prime}-\frac{\mu}{2}\right)\left\|\beta^{t+1}-\widehat{\beta}\right\|_{2}^{2}-\tau^{\prime} \frac{\log p}{n}\left\|\beta^{t+1}-\widehat{\beta}\right\|_{1}^{2} \leq\left(\overline{\mathcal{L}}_{n}\left(\beta^{t+1}\right)+\lambda\left\|\beta^{t+1}\right\|_{1}\right)-\left(\overline{\mathcal{L}}_{n}(\widehat{\beta})+\lambda\|\widehat{\beta}\|_{1}\right) .
$$

Inequality (68) gives an upper bound on the right-hand side of inequality (71). Combining the two inequalities, we then have

$$
\left(\alpha^{\prime}-\frac{\mu}{2}\right)\left\|\beta^{t+1}-\widehat{\beta}\right\|_{2}^{2}-\tau^{\prime} \frac{\log p}{n}\left\|\beta^{t+1}-\widehat{\beta}\right\|_{1}^{2} \leq \frac{\eta}{2}\left\|\beta^{t}-\widehat{\beta}\right\|_{2}^{2}-\frac{\eta}{2}\left\|\beta^{t+1}-\widehat{\beta}\right\|_{2}^{2}+\left(\tau^{\prime}+\tau^{\prime \prime}\right) \frac{4 R^{2} \log p}{n} .
$$

Hence,

$$
\begin{aligned}
\left\|\beta^{t+1}-\widehat{\beta}\right\|_{2}^{2} \leq & \frac{\eta / 2}{\alpha^{\prime}-\mu / 2+\eta / 2}\left\|\beta^{t}-\widehat{\beta}\right\|_{2}^{2} \\
& \quad+\frac{1}{\alpha^{\prime}-\mu / 2+\eta / 2}\left(\left(\tau^{\prime}+\tau^{\prime \prime}\right) \frac{4 R^{2} \log p}{n}+\tau^{\prime} \frac{\log p}{n}\left\|\beta^{t+1}-\widehat{\beta}\right\|_{1}^{2}\right) \\
\leq & \frac{\eta / 2}{\alpha^{\prime}-\mu / 2+\eta / 2}\left\|\beta^{t}-\widehat{\beta}\right\|_{2}^{2}+\frac{4\left(2 \tau^{\prime}+\tau^{\prime \prime}\right)}{\alpha^{\prime}-\mu / 2+\eta / 2} \cdot \frac{R^{2} \log p}{n} .
\end{aligned}
$$

Using the inductive hypothesis one more time and the scaling assumption (24), we conclude that

$$
\left\|\beta^{t+1}-\widehat{\beta}\right\|_{2}^{2} \leq \frac{\eta / 2}{\alpha^{\prime}-\mu / 2+\eta / 2} \cdot \frac{r^{2}}{4}+\frac{4\left(2 \tau^{\prime}+\tau^{\prime \prime}\right)}{\alpha^{\prime}-\mu / 2+\eta / 2} \cdot \frac{R^{2} \log p}{n} \leq \frac{r^{2}}{4},
$$

completing the induction.

LEMMA 6. Under the conditions of the theorem, suppose there exists a pair $(\bar{\eta}, T)$ such that

$$
\phi\left(\beta^{t}\right)-\phi(\widehat{\beta}) \leq \bar{\eta}, \quad \forall t \geq T .
$$

Then for any iteration $t \geq T$, we have

$$
\left\|\beta^{t}-\widehat{\beta}\right\|_{1} \leq 8 \sqrt{k}\left\|\beta^{t}-\widehat{\beta}\right\|_{2}+16 \sqrt{k}\left\|\widehat{\beta}-\beta^{*}\right\|_{2}+2 \cdot \min \left(\frac{2 \bar{\eta}}{\lambda}, R\right) .
$$


Proof. This proof is in fact a simplification of the argument used to prove Lemma 1 in Loh and Wainwright (2015), since by Lemma 5 and the assumption, we are guaranteed that

$$
\left\|\beta^{t}-\beta^{*}\right\|_{2} \leq\left\|\beta^{t}-\widehat{\beta}\right\|_{2}+\left\|\widehat{\beta}-\beta^{*}\right\|_{2} \leq \frac{r}{2}+\frac{r}{2}=r,
$$

so we may apply the RSC' condition (22) directly. Denoting $\Delta:=\beta^{t}-\beta^{*}$, we then have

$$
\begin{aligned}
\alpha^{\prime}\|\Delta\|_{2}^{2}-\tau^{\prime} \frac{\log p}{n}\|\Delta\|_{1}^{2} & \leq \mathcal{L}_{n}\left(\beta^{t}\right)-\mathcal{L}_{n}\left(\beta^{*}\right)-\left\langle\nabla \mathcal{L}_{n}\left(\beta^{*}\right), \Delta\right\rangle \\
& \leq \mathcal{L}_{n}\left(\beta^{t}\right)-\mathcal{L}_{n}\left(\beta^{*}\right)+\left\|\nabla \mathcal{L}_{n}\left(\beta^{*}\right)\right\|_{\infty} \cdot\|\Delta\|_{1} \\
& \leq \mathcal{L}_{n}\left(\beta^{t}\right)-\mathcal{L}_{n}\left(\beta^{*}\right)+\frac{\lambda}{8}\|\Delta\|_{1} .
\end{aligned}
$$

Furthermore, by assumption, we have

$$
\mathcal{L}_{n}\left(\beta^{t}\right)-\mathcal{L}_{n}\left(\beta^{*}\right)+\rho_{\lambda}\left(\beta^{t}\right)-\rho_{\lambda}\left(\beta^{*}\right) \leq \bar{\eta},
$$

which combined with inequality (72) implies that

$$
\alpha^{\prime}\|\Delta\|_{2}^{2}-\tau^{\prime} \frac{\log p}{n}\|\Delta\|_{1}^{2} \leq \rho_{\lambda}\left(\beta^{*}\right)-\rho_{\lambda}\left(\beta^{t}\right)+\bar{\eta}+\frac{\lambda}{8}\|\Delta\|_{1} .
$$

Note that if $\bar{\eta} \geq \frac{\lambda}{4}\|\Delta\|_{1}$, the desired inequality is trivial. Hence, we assume that $\bar{\eta} \leq \frac{\lambda}{4}\|\Delta\|_{1}$. In particular, inequality (74) implies that

$$
\begin{aligned}
\alpha^{\prime}\|\Delta\|_{2}^{2} & \leq \rho_{\lambda}\left(\beta^{*}\right)-\rho_{\lambda}\left(\beta^{t}\right)+\tau^{\prime} \frac{\log p}{n}\|\Delta\|_{1}^{2}+\frac{3 \lambda}{8}\|\Delta\|_{1} \\
& \leq \rho_{\lambda}\left(\beta^{*}\right)-\rho_{\lambda}\left(\beta^{t}\right)+2 \tau^{\prime} R \frac{\log p}{n}\|\Delta\|_{1}+\frac{3 \lambda}{8}\|\Delta\|_{1} \\
& \leq \rho_{\lambda}\left(\beta^{*}\right)-\rho_{\lambda}\left(\beta^{t}\right)+\frac{\lambda}{2}\|\Delta\|_{1} \\
& \leq \rho_{\lambda}\left(\beta^{*}\right)-\rho_{\lambda}\left(\beta^{t}\right)+\frac{\rho_{\lambda}(\Delta)}{2}+\frac{\mu}{4}\|\Delta\|_{2}^{2} \\
& \leq \rho_{\lambda}\left(\beta^{*}\right)-\rho_{\lambda}\left(\beta^{t}\right)+\frac{\rho_{\lambda}\left(\beta^{*}\right)+\rho_{\lambda}\left(\beta^{t}\right)}{2}+\frac{\mu}{4}\|\Delta\|_{2}^{2} \\
& \leq \frac{3}{2} \rho_{\lambda}\left(\beta^{*}\right)-\frac{1}{2} \rho_{\lambda}\left(\beta^{t}\right)+\frac{\mu}{4}\|\Delta\|_{2}^{2} .
\end{aligned}
$$

Hence, we have

$$
0 \leq\left(\alpha^{\prime}-\frac{\mu}{4}\right)\|\Delta\|_{2}^{2} \leq \frac{3}{2} \rho_{\lambda}\left(\beta^{*}\right)-\frac{1}{2} \rho_{\lambda}\left(\beta^{t}\right) .
$$

By Lemma 5 in Loh and Wainwright (2015), we then have

$$
\rho_{\lambda}\left(\beta^{*}\right)-\rho_{\lambda}\left(\beta^{t}\right) \leq 3 \rho_{\lambda}\left(\beta^{*}\right)-\rho_{\lambda}\left(\beta^{t}\right) \leq \lambda\left(3\left\|\Delta_{A}\right\|_{1}-\left\|\Delta_{A^{c}}\right\|_{1}\right),
$$


where $A$ indexes the top $k$ components of $\Delta$ in magnitude. Combining inequalities (74) and (75), we then have

$$
\alpha^{\prime}\|\Delta\|_{2}^{2}-\tau^{\prime} \frac{\log p}{n}\|\Delta\|_{1}^{2} \leq 3 \lambda\left\|\Delta_{A}\right\|_{1}-\lambda\left\|\Delta_{A^{c}}\right\|_{1}+\bar{\eta}+\frac{\lambda}{8}\|\Delta\|_{1},
$$

so

$$
\begin{aligned}
0 \leq \alpha^{\prime}\|\Delta\|_{2}^{2} & \leq 3 \lambda\left\|\Delta_{A}\right\|_{1}-\lambda\left\|\Delta_{A^{c}}\right\|_{1}+\bar{\eta}+\frac{\lambda}{8}\|\Delta\|_{1}+2 \tau^{\prime} R \frac{\log p}{n}\|\Delta\|_{1} \\
& \leq 3 \lambda\left\|\Delta_{A}\right\|_{1}-\lambda\left\|\Delta_{A^{c}}\right\|_{1}+\bar{\eta}+\frac{\lambda}{2}\|\Delta\|_{1} \\
& \leq \frac{7 \lambda}{2}\left\|\Delta_{A}\right\|_{1}-\frac{\lambda}{2}\left\|\Delta_{A^{c}}\right\|_{1}+\bar{\eta}
\end{aligned}
$$

Hence,

so

$$
\left\|\Delta_{A^{c}}\right\|_{1} \leq 7\left\|\Delta_{A}\right\|_{1}+\frac{2 \bar{\eta}}{\lambda}
$$

$$
\|\Delta\|_{1} \leq\left\|\Delta_{A}\right\|_{1}+\left\|\Delta_{A^{c}}\right\|_{1} \leq 8\left\|\Delta_{A}\right\|_{1}+\frac{2 \bar{\eta}}{\lambda} \leq 8 \sqrt{k}\|\Delta\|_{2}+\frac{2 \bar{\eta}}{\lambda} .
$$

Also, $\|\Delta\|_{1} \leq 2 R$, so we clearly have

$$
\|\Delta\|_{1} \leq 8 \sqrt{k}\|\Delta\|_{2}+2 \cdot \min \left(\frac{2 \bar{\eta}}{\lambda}, R\right) .
$$

Further note that by essentially the same argument, with inequality (73) replaced by

$$
\mathcal{L}_{n}(\widehat{\beta})-\mathcal{L}_{n}\left(\beta^{*}\right)+\rho_{\lambda}(\widehat{\beta})-\rho_{\lambda}\left(\beta^{*}\right) \leq 0,
$$

we have the inequality

$$
\left\|\widehat{\beta}-\beta^{*}\right\|_{1} \leq 8 \sqrt{k}\left\|\widehat{\beta}-\beta^{*}\right\|_{2}
$$

Combining inequalities (76) and (77) and using the triangle inequality then yields

$$
\begin{aligned}
\left\|\beta^{t}-\widehat{\beta}\right\|_{1} & \leq\left\|\widehat{\beta}-\beta^{*}\right\|_{1}+\left\|\beta^{t}-\beta^{*}\right\|_{1} \\
& \leq 8 \sqrt{k}\left(\left\|\widehat{\beta}-\beta^{*}\right\|_{2}+\left\|\beta^{t}-\beta^{*}\right\|_{2}\right)+2 \cdot \min \left(\frac{2 \bar{\eta}}{\lambda}, R\right) \\
& \leq 8 \sqrt{k}\left(\left\|\widehat{\beta}-\beta^{*}\right\|_{2}+\left\|\beta^{t}-\widehat{\beta}\right\|_{2}+\left\|\widehat{\beta}-\beta^{*}\right\|_{2}\right)+2 \cdot \min \left(\frac{2 \bar{\eta}}{\lambda}, R\right) \\
& =8 \sqrt{k}\left(\left\|\beta^{t}-\widehat{\beta}\right\|_{2}+2\left\|\widehat{\beta}-\beta^{*}\right\|_{2}\right)+2 \cdot \min \left(\frac{2 \bar{\eta}}{\lambda}, R\right)
\end{aligned}
$$

completing the proof. 


\section{APPENDIX D: PROOFS OF PROPOSITIONS IN SECTION 3.2}

In this Appendix, we provide the proofs of the technical propositions establishing sufficient conditions for statistical consistency of stationary points in Section 3.2.

D.1. Proof of Proposition 1. We have

$$
\left\|\nabla \mathcal{L}_{n}\left(\beta^{*}\right)\right\|_{\infty}=\left\|\frac{1}{n} \sum_{i=1}^{n} w\left(x_{i}\right) x_{i} \cdot \ell^{\prime}\left(\epsilon_{i} \cdot v\left(x_{i}\right)\right)\right\|_{\infty}
$$

Since $x_{i} \Perp \epsilon_{i}$ by assumption, the tower property of conditional expectation gives

$$
\mathbb{E}\left[w\left(x_{i}\right) x_{i} \cdot \ell^{\prime}\left(\epsilon_{i} \cdot v\left(x_{i}\right)\right)\right]=\mathbb{E}\left[\mathbb{E}\left[\ell^{\prime}\left(\epsilon_{i} \cdot v\left(x_{i}\right)\right) \mid x_{i}\right] \cdot w\left(x_{i}\right) x_{i}\right]
$$

Under condition (2a), the right-hand expression of equation (78) may be written as

$$
\mathbb{E}\left[\mathbb{E}\left[\ell^{\prime}\left(\epsilon_{i}\right) \mid x_{i}\right] \cdot w\left(x_{i}\right) x_{i}\right]=\mathbb{E}\left[\mathbb{E}\left[\ell^{\prime}\left(\epsilon_{i}\right)\right] \cdot w\left(x_{i}\right) x_{i}\right]=\mathbb{E}\left[\ell^{\prime}\left(\epsilon_{i}\right)\right] \cdot \mathbb{E}\left[w\left(x_{i}\right) x_{i}\right]=0 .
$$

If instead condition (2b) holds, the right-hand expression of equation (78) is clearly also equal to 0 .

Finally, note that since $\ell^{\prime}$ is bounded, the variables $\ell^{\prime}\left(\epsilon_{i} \cdot v\left(x_{i}\right)\right)$ are i.i.d. sub-Gaussian with parameter scaling with $\kappa_{1}$. By condition (1), the variables $w\left(x_{i}\right) x_{i}$ are also subGaussian. Hence, the desired bound holds by using standard concentration results for i.i.d. sums of products of sub-Gaussian variables.

D.2. Proof of Proposition 2. We begin with the outline of the main argument, with the proofs of supporting lemmas provided in subsequent subsections. The same general argument is used in the proofs of Propositions 3 and 4, as well.

D.2.1. Main argument. We have

$$
\begin{aligned}
\mathcal{T}\left(\beta_{1}, \beta_{2}\right) & :=\left\langle\nabla \mathcal{L}_{n}\left(\beta_{1}\right)-\nabla \mathcal{L}_{n}\left(\beta_{2}\right), \beta_{1}-\beta_{2}\right\rangle \\
& =\frac{1}{n} \sum_{i=1}^{n}\left(\ell^{\prime}\left(x_{i}^{T} \beta_{1}-y_{i}\right)-\ell^{\prime}\left(x_{i}^{T} \beta_{2}-y_{i}\right)\right) x_{i}^{T}\left(\beta_{1}-\beta_{2}\right) .
\end{aligned}
$$

Under the assumptions, equation (79) implies that

$$
\mathcal{T}\left(\beta_{1}, \beta_{2}\right) \geq \frac{1}{n} \sum_{i=1}^{n}\left(\ell^{\prime}\left(x_{i}^{T} \beta_{1}-y_{i}\right)-\ell^{\prime}\left(x_{i}^{T} \beta_{2}-y_{i}\right)\right) x_{i}^{T}\left(\beta_{1}-\beta_{2}\right) 1_{A_{i}}-\kappa_{2} \cdot \frac{1}{n} \sum_{i=1}^{n}\left(x_{i}^{T}\left(\beta_{1}-\beta_{2}\right)\right)^{2} 1_{A_{i}^{c}},
$$


where we set $\kappa_{2}=0$ in the case when $\ell$ is convex (but $\ell^{\prime \prime}$ does not necessarily exist everywhere), and the event $A_{i}$ is defined according to

$$
A_{i}:=\left\{\left|\epsilon_{i}\right| \leq \frac{T}{2}\right\} \cap\left\{\left|x_{i}^{T}\left(\beta_{1}-\beta_{2}\right)\right| \leq \frac{T}{8 r}\left\|\beta_{1}-\beta_{2}\right\|_{2}\right\} \cap\left\{\left|x_{i}^{T}\left(\beta_{2}-\beta^{*}\right)\right| \leq \frac{T}{4}\right\},
$$

for a parameter $T>0$, using the definition (16). Inequality (80) holds because when $\ell$ is convex, each summand in inequality (79) is always bounded below by 0 ; and when $\ell^{\prime \prime}$ exists and satisfies the bound (14), the mean value theorem gives

$$
\left(\ell^{\prime}\left(x_{i}^{T} \beta_{1}-y_{i}\right)-\ell^{\prime}\left(x_{i}^{T} \beta_{2}-y_{i}\right)\right) x_{i}^{T}\left(\beta_{1}-\beta_{2}\right)=\ell^{\prime \prime}\left(u_{i}\right)\left(x_{i}^{T}\left(\beta_{1}-\beta_{2}\right)\right)^{2} \geq-\kappa_{2}\left(x_{i}^{T}\left(\beta_{1}-\beta_{2}\right)\right)^{2},
$$

where $u_{i}$ is a point lying between $x_{i}^{T} \beta_{1}-y_{i}$ and $x_{i}^{T} \beta_{2}-y_{i}$.

Note that on $A_{i}$ and for $\left\|\beta_{1}-\beta^{*}\right\|_{2},\left\|\beta_{2}-\beta^{*}\right\|_{2} \leq r$, the triangle inequality gives

$$
\left|x_{i}^{T} \beta_{2}-y_{i}\right| \leq\left|x_{i}^{T}\left(\beta_{2}-\beta^{*}\right)\right|+\left|\epsilon_{i}\right| \leq T
$$

and

$$
\left|x_{i}^{T} \beta_{1}-y_{i}\right| \leq\left|x_{i}^{T}\left(\beta_{1}-\beta_{2}\right)\right|+\left|x_{i}^{T}\left(\beta_{2}-\beta^{*}\right)\right|+\left|\epsilon_{i}\right| \leq \frac{T}{4}+\frac{T}{4}+\frac{T}{2}=T .
$$

Hence, the mean value theorem implies that

$$
\ell^{\prime}\left(x_{i}^{T} \beta_{1}-y_{i}\right)-\ell^{\prime}\left(x_{i}^{T} \beta_{2}-y_{i}\right)=\ell^{\prime \prime}\left(u_{i}\right) x_{i}^{T}\left(\beta_{1}-\beta_{2}\right),
$$

for some $u_{i}$ with $\left|u_{i}\right| \leq T$. We then deduce from inequality (79) that

$$
\begin{aligned}
& \mathcal{T}\left(\beta_{1}, \beta_{2}\right) \geq \alpha_{T} \cdot \frac{1}{n} \sum_{i=1}^{n}\left(x_{i}^{T}\left(\beta_{1}-\beta_{2}\right)\right)^{2} 1_{A_{i}}-\kappa_{2} \cdot \frac{1}{n} \sum_{i=1}^{n}\left(x_{i}^{T}\left(\beta_{1}-\beta_{2}\right)\right)^{2} 1_{A_{i}^{c}} \\
&=\left(\alpha_{T}+\kappa_{2}\right) \cdot \frac{1}{n} \sum_{i=1}^{n}\left(x_{i}^{T}\left(\beta_{1}-\beta_{2}\right)\right)^{2} 1_{A_{i}}-\kappa_{2} \cdot \frac{1}{n} \sum_{i=1}^{n}\left(x_{i}^{T}\left(\beta_{1}-\beta_{2}\right)\right)^{2} \\
& \geq\left(\alpha_{T}+\kappa_{2}\right) \cdot \frac{1}{n} \sum_{i=1}^{n} \varphi_{T\left\|\beta_{1}-\beta_{2}\right\|_{2} / 8 r}\left(x_{i}^{T}\left(\beta_{1}-\beta_{2}\right)\right) \cdot \psi_{T / 2}\left(\epsilon_{i}\right) \cdot \psi_{T / 4}\left(x_{i}^{T}\left(\beta_{2}-\beta^{*}\right)\right) \\
& \quad-\kappa_{2} \cdot \frac{1}{n} \sum_{i=1}^{n}\left(x_{i}^{T}\left(\beta_{1}-\beta_{2}\right)\right)^{2} \\
&(82) \quad:=\left(\alpha_{T}+\kappa_{2}\right) \cdot f\left(\beta_{1}, \beta_{2}\right)-\kappa_{2} \cdot \widetilde{f}\left(\beta_{1}, \beta_{2}\right) .
\end{aligned}
$$

Here, we have defined the truncation functions

$$
\varphi_{t}(u)=\left\{\begin{array}{ll}
u^{2}, & \text { if }|u| \leq \frac{t}{2}, \\
(t-u)^{2}, & \text { if } \frac{t}{2} \leq|u| \leq t, \\
0, & \text { if }|u| \geq t,
\end{array} \quad \text { and } \quad \psi_{t}(u)= \begin{cases}1, & \text { if }|u| \leq \frac{t}{2} \\
2-\frac{2}{t}|u|, & \text { if } \frac{t}{2} \leq|u| \leq t \\
0, & \text { if }|u| \geq t,\end{cases}\right.
$$


as well as the functions

$$
\begin{aligned}
& f\left(\beta_{1}, \beta_{2}\right):=\frac{1}{n} \sum_{i=1}^{n} \varphi_{T\left\|\beta_{1}-\beta_{2}\right\|_{2} / 8 r}\left(x_{i}^{T}\left(\beta_{1}-\beta_{2}\right)\right) \cdot \psi_{T / 2}\left(\epsilon_{i}\right) \cdot \psi_{T / 4}\left(x_{i}^{T}\left(\beta_{2}-\beta^{*}\right)\right), \\
& \widetilde{f}\left(\beta_{1}, \beta_{2}\right):=\frac{1}{n} \sum_{i=1}^{n}\left(x_{i}^{T}\left(\beta_{1}-\beta_{2}\right)\right)^{2} .
\end{aligned}
$$

Note in particular that $\varphi_{t}$ and $\psi_{t}$ are $t$-Lipschitz and $\frac{2}{t}$-Lipschitz, respectively, and the truncation functions satisfy the bounds

$$
\varphi_{t}(u) \leq u^{2} \cdot 1\{|u| \leq t\}, \quad \text { and } \quad \psi_{t}(u) \leq 1\{|u| \leq t\}
$$

Note also that inequality (82) also implies the simple bound

$$
\left\langle\nabla \mathcal{L}_{n}\left(\beta_{1}\right)-\nabla \mathcal{L}_{n}\left(\beta_{2}\right), \beta_{1}-\beta_{2}\right\rangle \geq-\kappa_{2} \cdot \widetilde{f}\left(\beta_{1}, \beta_{2}\right) .
$$

We now define the sets

$$
B_{\delta}:=\left\{\left(\beta_{1}, \beta_{2}\right):\left\|\beta_{1}-\beta^{*}\right\|_{2},\left\|\beta_{2}-\beta^{*}\right\|_{2} \leq r, \frac{\left\|\beta_{1}-\beta_{2}\right\|_{1}}{\left\|\beta_{1}-\beta_{2}\right\|_{2}} \leq \delta, \beta_{1}, \beta_{2} \in \mathbb{B}_{1}(R)\right\},
$$

for a parameter $1 \leq \delta \leq c \sqrt{\frac{n}{\log p}}$. Let

$$
Z(\delta):=\sup _{\left(\beta_{1}, \beta_{2}\right) \in B_{\delta}}\left\{\frac{1}{\left\|\beta_{1}-\beta_{2}\right\|_{2}^{2}}\left|f\left(\beta_{1}, \beta_{2}\right)-\mathbb{E}\left[f\left(\beta_{1}, \beta_{2}\right)\right]\right|\right\}
$$

and

$$
\widetilde{Z}(\delta):=\sup _{\left(\beta_{1}, \beta_{2}\right) \in B_{\delta}}\left\{\frac{1}{\left\|\beta_{1}-\beta_{2}\right\|_{2}^{2}}\left|\widetilde{f}\left(\beta_{1}, \beta_{2}\right)-\mathbb{E}\left[\widetilde{f}\left(\beta_{1}, \beta_{2}\right)\right]\right|\right\} .
$$

With this notation, inequality (82) implies that for all $\left(\beta_{1}, \beta_{2}\right) \in B_{\delta}$, we have

$$
\begin{aligned}
& \frac{\mathcal{T}\left(\beta_{1}, \beta_{2}\right)}{\left\|\beta_{1}-\beta_{2}\right\|_{2}^{2}} \geq\left(\alpha_{T}+\kappa_{2}\right) \cdot \frac{\mathbb{E}\left[f\left(\beta_{1}, \beta_{2}\right)\right]}{\left\|\beta_{1}-\beta_{2}\right\|_{2}^{2}}-\kappa_{2} \cdot \frac{\mathbb{E}\left[\tilde{f}\left(\beta_{1}, \beta_{2}\right)\right]}{\left\|\beta_{1}-\beta_{2}\right\|_{2}^{2}}-\left(\alpha_{T}+\kappa_{2}\right) Z(\delta)-\kappa_{2} \widetilde{Z}(\delta) \\
& =\alpha_{T} \cdot \frac{\mathbb{E}\left[\widetilde{f}\left(\beta_{1}, \beta_{2}\right)\right]}{\left\|\beta_{1}-\beta_{2}\right\|_{2}^{2}}-\frac{\left(\alpha_{T}+\kappa_{2}\right)\left(\mathbb{E}\left[\tilde{f}\left(\beta_{1}, \beta_{2}\right)\right]-\mathbb{E}\left[f\left(\beta_{1}, \beta_{2}\right)\right]\right)}{\left\|\beta_{1}-\beta_{2}\right\|_{2}^{2}} \\
& \quad-\left(\alpha_{T}+\kappa_{2}\right) Z(\delta)-\kappa_{2} \widetilde{Z}(\delta) .
\end{aligned}
$$

The following lemma bounds the difference in expectations as a function of the truncation parameters. The proof is provided in Appendix D.2.2. 
LEMMA 7. We have the bound

$$
\mathbb{E}\left[\widetilde{f}\left(\beta_{1}, \beta_{2}\right)\right]-\mathbb{E}\left[f\left(\beta_{1}, \beta_{2}\right)\right] \leq c \sigma_{x}^{2}\left\|\beta_{1}-\beta_{2}\right\|_{2}^{2}\left(\epsilon_{T}^{1 / 2}+\exp \left(-\frac{c^{\prime} T^{2}}{\sigma_{x}^{2} r^{2}}\right)\right) .
$$

In particular, Lemma 7 implies that when inequality (17) holds, we have

$$
\left(\alpha_{T}+\kappa_{2}\right)\left(\mathbb{E}\left[\widetilde{f}\left(\beta_{1}, \beta_{2}\right)\right]-\mathbb{E}\left[f\left(\beta_{1}, \beta_{2}\right)\right]\right) \leq \frac{\alpha_{T}}{2} \cdot \mathbb{E}\left[\widetilde{f}\left(\beta_{1}, \beta_{2}\right)\right]
$$

since

$$
\mathbb{E}\left[\widetilde{f}\left(\beta_{1}, \beta_{2}\right)\right] \geq \lambda_{\min }\left(\Sigma_{x}\right) \cdot\left\|\beta_{1}-\beta_{2}\right\|_{2}^{2} .
$$

Then inequality (85) implies that

$$
\frac{\mathcal{T}\left(\beta_{1}, \beta_{2}\right)}{\left\|\beta_{1}-\beta_{2}\right\|_{2}^{2}} \geq \frac{\alpha_{T}}{2} \cdot \frac{\mathbb{E}\left[\widetilde{f}\left(\beta_{1}, \beta_{2}\right)\right]}{\left\|\beta_{1}-\beta_{2}\right\|_{2}^{2}}-\left(\alpha_{T}+\kappa_{2}\right) Z(\delta)-\kappa_{2} \widetilde{Z}(\delta) .
$$

We now focus on the terms $Z(\delta)$ and $\widetilde{Z}(\delta)$. Note that $\widetilde{f}\left(\beta_{1}, \beta_{2}\right)$ is a quadratic form in $\beta_{1}-\beta_{2}$, and for each unit vector $v \in \mathbb{R}^{p}$, the quantity $\frac{1}{n} \sum_{i=1}^{n}\left(x_{i}^{T} v\right)^{2}$ is an i.i.d. average of sub-exponential variables with parameter proportional to $\sigma_{x}^{2}$. Then by Lemmas 11 and 12 in Loh and Wainwright (2012), we have the bound

$$
\left|\widetilde{f}\left(\beta_{1}, \beta_{2}\right)-\mathbb{E}\left[\widetilde{f}\left(\beta_{1}, \beta_{2}\right)\right]\right| \leq t \sigma_{x}^{2}\left\|\beta_{1}-\beta_{2}\right\|_{2}^{2}+t \sigma_{x}^{2} \frac{\log p}{n}\left\|\beta_{1}-\beta_{2}\right\|_{1}^{2}, \quad \forall \beta_{1}, \beta_{2} \in \mathbb{R}^{p}
$$

with probability at least $1-c_{1} \exp \left(-c_{2} n t^{2}+c_{3} k \log p\right)$. In particular, since $\delta \leq c \sqrt{\frac{n}{\log p}}$, we may guarantee that

$$
\kappa_{2} \widetilde{Z}(\delta) \leq \frac{\alpha_{T}}{4} \cdot \frac{\mathbb{E}\left[\widetilde{f}\left(\beta_{1}, \beta_{2}\right)\right]}{\left\|\beta_{1}-\beta_{2}\right\|_{2}^{2}}
$$

w.h.p. Turning to $Z(\delta)$, we have the following lemma, proved in Appendix D.2.3:

Lemma 8. For some constants $c, c^{\prime}$, and $c^{\prime \prime}$, we have

$$
\mathbb{P}\left(Z(\delta) \geq c^{\prime \prime} \sigma_{x}\left(\frac{R T}{r^{2}}+\frac{\delta T}{r}\right) \sqrt{\frac{\log p}{n}}\right) \leq c \exp \left(-c^{\prime} \log p\right) .
$$

Combining inequalities (88) and (89) with inequality (86), we then have

$$
\frac{\mathcal{T}\left(\beta_{1}, \beta_{2}\right)}{\left\|\beta_{1}-\beta_{2}\right\|_{2}^{2}} \geq \frac{\alpha_{T}}{4} \cdot \frac{\mathbb{E}\left[\widetilde{f}\left(\beta_{1}, \beta_{2}\right)\right]}{\left\|\beta_{1}-\beta_{2}\right\|_{2}^{2}}-\left(\alpha_{T}+\kappa_{2}\right) c^{\prime \prime} \sigma_{x}\left(\frac{R T}{r^{2}}+\frac{\delta T}{r}\right) \sqrt{\frac{\log p}{n}},
$$


with probability at least $1-c_{1} \exp \left(-c_{2} \log p\right)$. Let $n \succsim R^{2} \log p$ be chosen such that

$$
\left(\alpha_{T}+\kappa_{2}\right) c^{\prime \prime} \sigma_{x} \cdot \frac{R T}{r^{2}} \sqrt{\frac{\log p}{n}} \leq \frac{\alpha_{T}}{8} \cdot \frac{\mathbb{E}\left[\tilde{f}\left(\beta_{1}, \beta_{2}\right)\right]}{\left\|\beta_{1}-\beta_{2}\right\|_{2}^{2}} .
$$

Then inequality (90) implies that

$$
\frac{\mathcal{T}\left(\beta_{1}, \beta_{2}\right)}{\left\|\beta_{1}-\beta_{2}\right\|_{2}^{2}} \geq \frac{\alpha_{T}}{8} \cdot \frac{\mathbb{E}\left[\tilde{f}\left(\beta_{1}, \beta_{2}\right)\right]}{\left\|\beta_{1}-\beta_{2}\right\|_{2}^{2}}-\frac{c^{\prime \prime}\left(\alpha_{T}+\kappa_{2}\right) \sigma_{x} T}{r} \delta \sqrt{\frac{\log p}{n}} .
$$

We now extend inequality (91) to a bound that holds uniformly over the domain, with $\delta$ replaced by $\frac{\left\|\beta_{1}-\beta_{2}\right\|_{1}}{\left\|\beta_{1}-\beta_{2}\right\|_{2}}$. This is accomplished via a peeling argument in the proof of the following lemma:

Lemma 9. Fix $c_{0}>0$, and let

$$
\begin{aligned}
& \mathcal{D}:=\left\{\beta_{1}, \beta_{2} \in \mathbb{B}_{1}(R):\left\|\beta_{1}-\beta^{*}\right\|_{2},\left\|\beta_{2}-\beta^{*}\right\|_{2} \leq r\right. \\
& \left.\quad \text { and } \frac{\left\|\beta_{1}-\beta_{2}\right\|_{1}}{\left\|\beta_{1}-\beta_{2}\right\|_{2}} \leq \frac{c_{0} \alpha_{T} \lambda_{\min }\left(\Sigma_{x}\right) r}{\sigma_{x}\left(\alpha_{T}+\kappa_{2}\right) T} \sqrt{\frac{n}{\log p}}\right\} .
\end{aligned}
$$

With probability at least $1-c_{1}^{\prime} \exp \left(-c_{2}^{\prime} \log p\right)$, the following inequality holds uniformly over all $\beta_{1}, \beta_{2} \in \mathcal{D}$ :

$$
\begin{aligned}
\frac{\mathcal{T}\left(\beta_{1}, \beta_{2}\right)}{\left\|\beta_{1}-\beta_{2}\right\|_{2}^{2}} & \geq \alpha_{T} \cdot \frac{\lambda_{\min }\left(\Sigma_{x}\right)}{8}-\frac{c^{\prime \prime}\left(\alpha_{T}+\kappa_{2}\right) \sigma_{x} T}{c_{0} r} \frac{\left\|\beta_{1}-\beta_{2}\right\|_{1}}{\left\|\beta_{1}-\beta_{2}\right\|_{2}} \sqrt{\frac{\log p}{n}} \\
& \geq \alpha_{T} \cdot \frac{\lambda_{\min }\left(\Sigma_{x}\right)}{16}-\frac{c^{\prime \prime \prime}\left(\alpha_{T}+\kappa_{2}\right)^{2} \sigma_{x}^{2} T^{2}}{c_{0}^{2} r^{2}} \frac{\log p}{n} \frac{\left\|\beta_{1}-\beta_{2}\right\|_{1}^{2}}{\left\|\beta_{1}-\beta_{2}\right\|_{2}^{2}}
\end{aligned}
$$

The proof of Lemma 9 is provided in Appendix D.2.4.

Finally, note that inequality (87) implies the bound

$$
\widetilde{f}\left(\beta_{1}, \beta_{2}\right) \leq \alpha^{\prime}\left\|\beta_{1}-\beta_{2}\right\|_{2}^{2}+\tau^{\prime} \frac{\log p}{n}\left\|\beta_{1}-\beta_{2}\right\|_{1}^{2}, \quad \forall \beta_{1}, \beta_{2} \in \mathbb{R}^{p} .
$$

Together with inequality (84), we then see that for a proper choice of the constant $c_{0}$, we have

$$
\begin{aligned}
\mathcal{T}\left(\beta_{1}, \beta_{2}\right) & \geq-\kappa_{2}\left(\alpha^{\prime}\left\|\beta_{1}-\beta_{2}\right\|_{2}^{2}-\tau^{\prime} \frac{\log p}{n}\left\|\beta_{1}-\beta_{2}\right\|_{1}^{2}\right) \\
& \geq \alpha_{T} \cdot \frac{\lambda_{\min }\left(\Sigma_{x}\right)}{16}\left\|\beta_{1}-\beta_{2}\right\|_{2}^{2}-\frac{c^{\prime \prime \prime}\left(\alpha_{T}+\kappa_{2}\right)^{2} \sigma_{x}^{2} T^{2}}{c_{0}^{2} r^{2}} \frac{\log p}{n}\left\|\beta_{1}-\beta_{2}\right\|_{1}^{2}
\end{aligned}
$$

whenever $\frac{\left\|\beta_{1}-\beta_{2}\right\|_{1}}{\left\|\beta_{1}-\beta_{2}\right\|_{2}}>\frac{c_{0} r}{c^{\prime \prime \prime}\left(\alpha_{T}+\kappa_{2}\right) \sigma_{x} \tau} \sqrt{\frac{n}{\log p}}$. Combined with Lemma 9, inequality (94) implies the desired condition (10). 
D.2.2. Proof of Lemma \%. Note that

$$
\begin{aligned}
& \mathbb{E}\left[\left(x_{i}^{T}\left(\beta_{1}-\beta_{2}\right)\right)^{2}\right]-\mathbb{E}\left[f\left(\beta_{1}, \beta_{2}\right)\right] \leq \mathbb{E} {\left[\left(x_{i}^{T}\left(\beta_{1}-\beta_{2}\right)\right)^{2} 1\left\{\left|x_{i}^{T}\left(\beta_{1}-\beta_{2}\right)\right| \geq \frac{T}{8 r}\left\|\beta_{1}-\beta_{2}\right\|_{2}\right\}\right] } \\
&+\mathbb{E}\left[\left(x_{i}^{T}\left(\beta_{1}-\beta_{2}\right)\right)^{2} 1\left\{\left|\epsilon_{i}\right| \geq \frac{T}{2}\right\}\right] \\
&+\mathbb{E}\left[\left(x_{i}^{T}\left(\beta_{1}-\beta_{2}\right)\right)^{2} 1\left\{\left|x_{i}^{T}\left(\beta_{2}-\beta^{*}\right)\right| \geq \frac{T}{4}\right\}\right] .
\end{aligned}
$$

Applying the Cauchy-Schwarz inequality, we have bounds of the form

$$
\mathbb{E}\left[\left(x_{i}^{T}\left(\beta_{1}-\beta_{2}\right)\right)^{2} 1_{E_{i}}\right] \leq \mathbb{E}\left[\left(x_{i}^{T}\left(\beta_{1}-\beta_{2}\right)\right)^{4}\right]^{1 / 2} \cdot \mathbb{E}\left[1_{E_{i}}\right]^{1 / 2} \leq c \sigma_{x}^{2}\left\|\beta_{1}-\beta_{2}\right\|_{2}^{2} \cdot\left(\mathbb{P}\left(E_{i}\right)\right)^{1 / 2},
$$

where the second inequality holds because of the assumption that $x_{i}$ is sub-Gaussian with parameter $\sigma_{x}$.

Furthermore, note that

$$
\mathbb{P}\left(\left|x_{i}^{T}\left(\beta_{2}-\beta^{*}\right)\right| \geq \frac{T}{4}\right) \leq c \exp \left(-\frac{c^{\prime} T^{2}}{\sigma_{x}^{2} r^{2}}\right)
$$

since $x_{i}$ is sub-Gaussian and $\left\|\beta_{2}-\beta^{*}\right\|_{2} \leq r$ by assumption. Finally, we have

$$
\mathbb{P}\left(\left|x_{i}^{T}\left(\beta_{1}-\beta_{2}\right)\right| \geq \frac{T}{8 r}\left\|\beta_{1}-\beta_{2}\right\|_{2}\right) \leq c \exp \left(-\frac{c^{\prime} T^{2}}{\sigma_{x}^{2} r^{2}}\right)
$$

also by sub-Gaussianity of $x_{i}$. Combining these bounds with inequality (95) then implies the desired result.

D.2.3. Proof of Lemma 8. We first bound $\mathbb{E}[Z(\delta)]$. Following the argument in the proof of Lemma 11 of Loh and Wainwright (2015), we have

$\mathbb{E}[Z(\delta)] \leq 2 \sqrt{\frac{\pi}{2}} \mathbb{E}\left[\sup _{\left(\beta_{1}, \beta_{2}\right) \in B_{\delta}} \frac{1}{\|\Delta\|_{2}^{2}}\left|\frac{1}{n} \sum_{i=1}^{n} g_{i} \cdot \varphi_{\frac{T\|\Delta\|_{2}}{8 r}}\left(x_{i}^{T}\left(\beta_{1}-\beta_{2}\right)\right) \psi_{\frac{T}{2}}\left(\epsilon_{i}\right) \psi_{\frac{T}{4}}\left(x_{i}^{T}\left(\beta_{2}-\beta^{*}\right)\right)\right|\right]$,

where we denote $\Delta:=\beta_{1}-\beta_{2}$, and the $g_{i}$ 's are i.i.d. standard Gaussians. Define

$$
Z_{\beta_{1}, \beta_{2}}:=\frac{1}{\|\Delta\|_{2}^{2}} \cdot \frac{1}{n} \sum_{i=1}^{n} g_{i} \cdot \varphi_{\frac{T\|\Delta\|_{2}}{8 r}}\left(x_{i}^{T}\left(\beta_{1}-\beta_{2}\right)\right) \psi_{\frac{T}{2}}\left(\epsilon_{i}\right) \psi_{\frac{T}{4}}\left(x_{i}^{T}\left(\beta_{2}-\beta^{*}\right)\right),
$$

and note that conditioned on the $x_{i}$ 's and $\epsilon_{i}$ 's, each variable $Z_{\beta_{1}, \beta_{2}}$ is a Gaussian process. Furthermore, for distinct pairs $\left(\beta_{1}, \beta_{2}\right)$ and $\left(\beta_{1}^{\prime}, \beta_{2}^{\prime}\right)$, we have

$$
\operatorname{var}\left(Z_{\beta_{1}, \beta_{2}}-Z_{\beta_{1}^{\prime}, \beta_{2}^{\prime}}\right) \leq 2 \operatorname{var}\left(Z_{\beta_{1}, \beta_{2}}-Z_{\beta_{2}^{\prime}+\Delta, \beta_{2}^{\prime}}\right)+2 \operatorname{var}\left(Z_{\beta_{2}+\Delta, \beta_{2}^{\prime}}-Z_{\beta_{1}^{\prime}, \beta_{2}^{\prime}}\right)
$$


Continuing to condition on the $x_{i}$ 's and $\epsilon_{i}{ }^{\prime}$ s, and denoting $\Delta^{\prime}:=\beta_{1}^{\prime}-\beta_{2}^{\prime}$, note that

$$
\begin{aligned}
\operatorname{var}\left(Z_{\beta_{2}^{\prime}+\Delta, \beta_{2}^{\prime}}-Z_{\beta_{1}^{\prime}, \beta_{2}^{\prime}}\right)=\frac{1}{n^{2}} \sum_{i=1}^{n} \psi_{\frac{T}{2}}^{2}\left(\epsilon_{i}\right) \psi_{\frac{T}{4}}^{2}\left(x_{i}^{T}\left(\beta_{2}^{\prime}-\beta^{*}\right)\right) \\
\cdot\left(\frac{1}{\|\Delta\|_{2}^{2}} \varphi_{\frac{T\|\Delta\|_{2}}{8 r}}\left(x_{i}^{T} \Delta\right)-\frac{1}{\left\|\Delta^{\prime}\right\|_{2}^{2}} \varphi_{\frac{T\left\|\Delta^{\prime}\right\|_{2}}{8 r}}\left(x_{i}^{T} \Delta^{\prime}\right)\right)^{2}
\end{aligned}
$$

Furthermore, $\varphi$ satisfies the homogeneity property that

$$
\frac{1}{c^{2}} \cdot \varphi_{c t}(c u)=\varphi_{t}(u), \quad \forall c>0
$$

Hence, inequality (96) implies that

$$
\begin{aligned}
\operatorname{var}\left(Z_{\beta_{2}^{\prime}+\Delta, \beta_{2}^{\prime}}-Z_{\beta_{1}^{\prime}, \beta_{2}^{\prime}}\right) & \leq \frac{1}{n^{2}} \sum_{i=1}^{n} \frac{1}{\|\Delta\|_{2}^{4}}\left(\varphi_{\frac{T\|\Delta\|_{2}}{8 r}}\left(x_{i}^{T} \Delta\right)-\varphi_{\frac{T\|\Delta\|_{2}}{8 r}}\left(x_{i}^{T} \Delta^{\prime} \cdot \frac{\|\Delta\|_{2}}{\left\|\Delta^{\prime}\right\|_{2}}\right)\right)^{2} \\
& \leq \frac{1}{n^{2}} \sum_{i=1}^{n} \frac{1}{\|\Delta\|_{2}^{4}} \cdot \frac{T^{2}\|\Delta\|_{2}^{2}}{64 r^{2}}\left(x_{i}^{T} \Delta-x_{i}^{T} \Delta^{\prime} \cdot \frac{\|\Delta\|_{2}}{\left\|\Delta^{\prime}\right\|_{2}}\right)^{2} \\
& =\frac{1}{n^{2}} \sum_{i=1}^{n} \frac{T^{2}}{64 r^{2}}\left(\frac{x_{i}^{T} \Delta}{\|\Delta\|_{2}}-\frac{x_{i}^{T} \Delta^{\prime}}{\left\|\Delta^{\prime}\right\|_{2}}\right)^{2}
\end{aligned}
$$

where the second inequality uses the Lipschitz property of $\varphi$. Similarly, we may calculate

$$
\begin{aligned}
\operatorname{var}\left(Z_{\beta_{1}, \beta_{2}}-Z_{\beta_{2}^{\prime}+\Delta, \beta_{2}^{\prime}}\right) & =\frac{1}{n^{2}} \sum_{i=1}^{n} \frac{1}{\|\Delta\|_{2}^{4}} \psi_{\frac{T}{2}}^{2}\left(\epsilon_{i}\right) \\
& \cdot \varphi_{\frac{T\|\Delta\|_{2}}{8 r}}^{2}\left(x_{i}^{T} \Delta\right)\left(\psi_{\frac{T}{4}}\left(x_{i}^{T}\left(\beta_{2}-\beta^{*}\right)\right)-\psi_{\frac{T}{4}}\left(x_{i}^{T}\left(\beta_{2}^{\prime}-\beta^{*}\right)\right)\right)^{2}
\end{aligned}
$$

Using the fact that $\psi_{T / 4}$ is $\frac{8}{T}$-Lipschitz and $\left\|\varphi_{T\|\Delta\|_{2} /(8 r)}\right\|_{\infty} \leq \frac{T^{2}\|\Delta\|_{2}^{2}}{256 r^{2}}$, inequality (97) implies that

$$
\begin{aligned}
\operatorname{var}\left(Z_{\beta_{1}, \beta_{2}}-Z_{\beta_{2}^{\prime}+\Delta, \beta_{2}^{\prime}}\right) & \leq \frac{1}{n^{2}} \sum_{i=1}^{n} \frac{T^{4}}{256^{2} r^{4}} \cdot \frac{64}{T^{2}}\left(x_{i}^{T}\left(\beta_{2}-\beta_{2}^{\prime}\right)\right)^{2} \\
& =\frac{1}{n^{2}} \sum_{i=1}^{n} \frac{T^{2}}{32^{2} r^{4}}\left(x_{i}^{T}\left(\beta_{2}-\beta_{2}^{\prime}\right)\right)^{2}
\end{aligned}
$$

If we define the second Gaussian process

$$
Y_{\beta_{1}, \beta_{2}}:=\frac{T}{16 r^{2}} \cdot \frac{1}{n} \sum_{i=1}^{n} g_{i}^{\prime} \cdot x_{i}^{T} \beta_{2}+\frac{T}{4 r} \cdot \frac{1}{n} \sum_{i=1}^{n} g_{i}^{\prime \prime} \cdot \frac{x_{i}^{T}\left(\beta_{1}-\beta_{2}\right)}{\left\|\beta_{1}-\beta_{2}\right\|_{2}},
$$


where $g_{i}^{\prime}$ and $g_{i}^{\prime \prime}$ are independent standard Gaussians, the above calculation implies that

$$
\operatorname{var}\left(Z_{\beta_{1}, \beta_{2}}-Z_{\beta_{1}^{\prime}, \beta_{2}^{\prime}}\right) \leq \operatorname{var}\left(Y_{\beta_{1}, \beta_{2}}-Y_{\beta_{1}^{\prime}, \beta_{2}^{\prime}}\right)
$$

Hence, Lemma 14 in Loh and Wainwright (2015) implies that

$$
\mathbb{E}\left[\sup _{\left(\beta_{1}, \beta_{2}\right) \in B_{\delta}} Z_{\beta_{1}, \beta_{2}}\right] \leq 2 \cdot \mathbb{E}\left[\sup _{\left(\beta_{1}, \beta_{2}\right) \in B_{\delta}} Y_{\beta_{1}, \beta_{2}}\right],
$$

where the expectations are no longer conditional. By an argument from Ledoux and Talagrand (1991), we also have

$$
\mathbb{E}\left[\sup _{\left(\beta_{1}, \beta_{2}\right) \in B_{\delta}}\left|Z_{\beta_{1}, \beta_{2}}\right|\right] \leq \mathbb{E}\left[\left|Z_{\beta_{1}^{\prime}, \beta_{2}^{\prime}}\right|\right]+2 \cdot \mathbb{E}\left[\sup _{\left(\beta_{1}, \beta_{2}\right) \in B_{\delta}} Z_{\beta_{1}, \beta_{2}}\right],
$$

for any fixed $\left(\beta_{1}^{\prime}, \beta_{2}^{\prime}\right) \in B_{\delta}$. Furthermore,

$$
\mathbb{E}\left[\left|Z_{\beta_{1}^{\prime}, \beta_{2}^{\prime}}\right|\right] \leq c \sqrt{\operatorname{var}\left(Z_{\beta_{1}^{\prime}, \beta_{2}^{\prime}}\right)} \leq \frac{c T^{2}}{256 r^{2}} \cdot \frac{1}{\sqrt{n}},
$$

by conditioning on the $x_{i}$ 's and using the bounds on $\varphi$ and $\psi$. We also have the bound

$$
\begin{aligned}
\mathbb{E}\left[\sup _{\left(\beta_{1}, \beta_{2}\right) \in B_{\delta}} Y_{\beta_{1}, \beta_{2}}\right] & \leq \frac{R T}{16 r^{2}} \cdot \mathbb{E}\left[\left\|\frac{1}{n} \sum_{i=1}^{n} g_{i}^{\prime} x_{i}\right\|_{\infty}\right]+\frac{\delta T}{4 r} \cdot \mathbb{E}\left[\left\|\frac{1}{n} \sum_{i=1}^{n} g_{i}^{\prime \prime} x_{i}\right\|_{\infty}\right] \\
& \leq c \sigma_{x}\left(\frac{R T}{r^{2}}+\frac{\delta T}{r}\right) \sqrt{\frac{\log p}{n}}
\end{aligned}
$$

Hence,

$$
\mathbb{E}[Z(\delta)] \leq c^{\prime} \sigma_{x}\left(\frac{R T}{r^{2}}+\frac{\delta T}{r}\right) \sqrt{\frac{\log p}{n}} .
$$

Further note that for $\left(\beta_{1}, \beta_{2}\right) \in B_{\delta}$, each summand in $f\left(\beta_{1}, \beta_{2}\right)$ lies in the interval $\left[0, \frac{T^{2}}{64 r^{2}}\right]$. Hence, by the bounded differences inequality, we have

$$
\mathbb{P}(|Z(\delta)-\mathbb{E}[Z(\delta)]| \geq t) \leq c \exp \left(-\frac{c^{\prime} r^{2}}{T^{2}} n t^{2}\right)
$$

Combining inequalities (98) and (99) then gives the desired result. 
D.2.4. Proof of Lemma 9. We parallel the peeling argument constructed in the proof of Lemma 11 in Loh and Wainwright (2015). Define the event

$$
\mathcal{E}:=\left\{\text { inequality }(92) \text { holds } \forall \beta_{1}, \beta_{2} \in \mathcal{D}\right\},
$$

and define the functions

$$
\begin{aligned}
\widetilde{h}\left(\beta_{1}, \beta_{2} ; X\right) & :=\alpha_{T} \cdot \frac{\lambda_{\min }\left(\Sigma_{x}\right)}{8}-\frac{\mathcal{T}\left(\beta_{1}, \beta_{2}\right)}{\left\|\beta_{1}-\beta_{2}\right\|_{2}^{2}}, \\
g(\delta) & :=\frac{c^{\prime \prime}\left(\alpha_{T}+\kappa_{2}\right) \sigma_{x} T}{2 c_{0} r} \cdot \delta \sqrt{\frac{\log p}{n}} \\
h\left(\beta_{1}, \beta_{2}\right) & :=\frac{\left\|\beta_{1}-\beta_{2}\right\|_{1}}{\left\|\beta_{1}-\beta_{2}\right\|_{2}} .
\end{aligned}
$$

By inequality (91), we have

$$
\mathbb{P}\left(\sup _{\substack{\left.\beta_{1}, \beta_{2}\right) \in \mathcal{D}: \\ h\left(\beta_{1}, \beta_{2}\right) \leq \delta}} \tilde{h}\left(\beta_{1}, \beta_{2} ; X\right) \geq g(\delta)\right) \leq c_{1} \exp \left(-c_{2} \log p\right),
$$

for any $1 \leq \delta \leq \frac{c_{0} \alpha_{T} \lambda_{\min }\left(\Sigma_{x}\right) r}{\sigma_{x}\left(\alpha_{T}+\kappa_{2}\right) T} \sqrt{\frac{n}{\log p}}$. Since $\frac{\left\|\beta_{1}-\beta_{2}\right\|_{1}}{\left\|\beta_{1}-\beta_{2}\right\|_{2}} \geq 1$, we have

$$
1 \leq h\left(\beta_{1}, \beta_{2}\right) \leq \frac{c_{0} \alpha_{T} \lambda_{\min }\left(\Sigma_{x}\right)}{\sigma_{x}\left(\alpha_{T}+\kappa_{2}\right) T} \sqrt{\frac{n}{\log p}},
$$

over the region of interest. For each integer $m \geq 1$, define the set

$$
V_{m}:=\left\{\left(\beta_{1}, \beta_{2}\right): 2^{m-1} \mu \leq g\left(h\left(\beta_{1}, \beta_{2}\right)\right) \leq 2^{m} \mu\right\} \cap \mathcal{D},
$$

where $\mu:=\frac{c \sigma_{x} T}{c_{0} r} \sqrt{\frac{\log p}{n}}$. A union bound gives

$$
\mathbb{P}\left(\mathcal{E}^{c}\right) \leq \sum_{m=1}^{M} \mathbb{P}\left\{\exists\left(\beta_{1}, \beta_{2}\right) \in V_{m}: \widetilde{h}\left(\beta_{1}, \beta_{2} ; X\right) \geq 2 g\left(h\left(\beta_{1}, \beta_{2}\right)\right)\right\},
$$

where $M:=\left\lceil\log \left(c \sqrt{\frac{n}{\log p}}\right)\right\rceil$. Then

$$
\mathbb{P}\left(\mathcal{E}^{c}\right) \leq \sum_{m=1}^{M} \mathbb{P}\left(\sup _{\substack{\left(\beta_{1}, \beta_{2}\right) \in \mathcal{D}: \\ h\left(\beta_{1}, \beta_{2}\right) \leq g^{-1}\left(2^{m} \mu\right)}} \tilde{h}\left(\beta_{1}, \beta_{2} ; X\right) \geq 2^{m} \mu\right) \leq M \cdot c_{1} \exp \left(-c_{2} \log p\right),
$$

using inequality (100). Hence,

$$
\mathbb{P}\left(\mathcal{E}^{c}\right) \leq c_{1} \exp \left(-c_{2} \log p+\log \log \left(\frac{n}{\log p}\right)\right) \leq c_{1}^{\prime} \exp \left(-c_{2}^{\prime} \log p\right) .
$$

Inequality (93) holds by applying the arithmetic mean-geometric mean inequality to inequality (92). 
D.3. Proof of Proposition 3. Again defining $\mathcal{T}\left(\beta_{1}, \beta_{2}\right)$ as in equation (79), we have

$$
\mathcal{T}\left(\beta_{1}, \beta_{2}\right)=\frac{1}{n} \sum_{i=1}^{n} w\left(x_{i}\right)\left(\ell^{\prime}\left(x_{i}^{T} \beta_{1}-y_{i}\right)-\ell^{\prime}\left(x_{i}^{T} \beta_{2}-y_{i}\right)\right) x_{i}^{T}\left(\beta_{1}-\beta_{2}\right) .
$$

Defining the event $A_{i}$ as in equation (81), inequality (101) implies that

$$
\begin{aligned}
\mathcal{T}\left(\beta_{1}, \beta_{2}\right) & \geq \frac{1}{n} \sum_{i=1}^{n} w\left(x_{i}\right)\left(\ell^{\prime}\left(x_{i}^{T} \beta_{1}-y_{i}\right)-\ell^{\prime}\left(x_{i}^{T} \beta_{2}-y_{i}\right)\right) x_{i}^{T}\left(\beta_{1}-\beta_{2}\right) \\
& \geq \alpha_{T} \cdot \frac{1}{n} \sum_{i=1}^{n} w\left(x_{i}\right)\left(x_{i}^{T}\left(\beta_{1}-\beta_{2}\right)\right)^{2} 1_{A_{i}}-\kappa_{2} \cdot \frac{1}{n} \sum_{i=1}^{n} w\left(x_{i}\right)\left(x_{i}^{T}\left(\beta_{1}-\beta_{2}\right)\right)^{2} 1_{A_{i}^{c}} \\
& =\left(\alpha_{T}+\kappa_{2}\right) \cdot \frac{1}{n} \sum_{i=1}^{n} w\left(x_{i}\right)\left(x_{i}^{T}\left(\beta_{1}-\beta_{2}\right)\right)^{2} 1_{A_{i}}-\kappa_{2} \cdot \frac{1}{n} \sum_{i=1}^{n} w\left(x_{i}\right)\left(x_{i}^{T}\left(\beta_{1}-\beta_{2}\right)\right)^{2} .
\end{aligned}
$$

Note that $w\left(x_{i}\right) x_{i}$ is a sub-Gaussian vector with parameter $c b_{0}$. Defining the truncation functions $\varphi$ and $\psi$ as in equations (83), we then have

$$
\mathcal{T}\left(\beta_{1}, \beta_{2}\right) \geq\left(\alpha_{T}+\kappa_{2}\right) \cdot f\left(\beta_{1}, \beta_{2}\right)-\kappa_{2} \cdot \widetilde{f}\left(\beta_{1}, \beta_{2}\right),
$$

as in inequality (82), where

$$
\begin{aligned}
f\left(\beta_{1}, \beta_{2}\right) & :=\frac{1}{n} \sum_{i=1}^{n} w\left(x_{i}\right) \cdot \varphi_{T\left\|\beta_{1}-\beta_{2}\right\|_{2} / 8 r}\left(x_{i}^{T}\left(\beta_{1}-\beta_{2}\right)\right) \cdot \psi_{T / 2}\left(\epsilon_{i}\right) \cdot \psi_{T / 4}\left(x_{i}^{T}\left(\beta_{2}-\beta^{*}\right)\right), \\
\widetilde{f}\left(\beta_{1}, \beta_{2}\right) & :=\frac{1}{n} \sum_{i=1}^{n} w\left(x_{i}\right)\left(x_{i}^{T}\left(\beta_{1}-\beta_{2}\right)\right)^{2} .
\end{aligned}
$$

We first obtain an analog of Lemma 7, as follows:

LEMma 10. We have the bound

$$
\mathbb{E}\left[\widetilde{f}\left(\beta_{1}, \beta_{2}\right)\right]-\mathbb{E}\left[f\left(\beta_{1}, \beta_{2}\right)\right] \leq c b_{0} \sigma_{x}^{2}\left\|\beta_{1}-\beta_{2}\right\|_{2}^{2}\left(\epsilon_{T}^{1 / 2}+\exp \left(-\frac{c^{\prime} T}{\sigma_{x} r}\right)\right) .
$$

Proof. We have

$$
\begin{aligned}
& \mathbb{E}\left[w\left(x_{i}\right)\left(x_{i}^{T}\left(\beta_{1}-\beta_{2}\right)\right)^{2}\right]-\mathbb{E}[\left.f\left(\beta_{1}, \beta_{2}\right)\right] \\
& \leq \mathbb{E} {\left[w\left(x_{i}\right)\left(x_{i}^{T}\left(\beta_{1}-\beta_{2}\right)\right)^{2} 1\left\{\left|x_{i}^{T}\left(\beta_{1}-\beta_{2}\right)\right| \geq \frac{T}{8 r}\left\|\beta_{1}-\beta_{2}\right\|_{2}\right\}\right] } \\
&+ \mathbb{E}\left[w\left(x_{i}\right)\left(x_{i}^{T}\left(\beta_{1}-\beta_{2}\right)\right)^{2} 1\left\{\left|\epsilon_{i}\right| \geq \frac{T}{2}\right\}\right] \\
&+\mathbb{E}\left[w\left(x_{i}\right)\left(x_{i}^{T}\left(\beta_{1}-\beta_{2}\right)\right)^{2} 1\left\{\left|x_{i}^{T}\left(\beta_{2}-\beta^{*}\right)\right| \geq \frac{T}{4}\right\}\right] .
\end{aligned}
$$


Applying the Cauchy-Schwarz inequality to each term, the right-hand side of inequality (102) is then upper-bounded by

$$
\begin{gathered}
\mathbb{E}\left[w^{2}\left(x_{i}\right)\left(x_{i}^{T}\left(\beta_{1}-\beta_{2}\right)\right)^{4}\right]^{1 / 2} \cdot\left\{\mathbb{P}\left(\left|x_{i}^{T}\left(\beta_{1}-\beta_{2}\right)\right| \geq \frac{T}{8 r}\left\|\beta_{1}-\beta_{2}\right\|_{2}\right)^{1 / 2}\right. \\
\left.\quad+\mathbb{P}\left(\left|\epsilon_{i}\right| \geq \frac{T}{2}\right)^{1 / 2}+\mathbb{P}\left(\left|x_{i}^{T}\left(\beta_{2}-\beta^{*}\right)\right| \geq \frac{T}{4}\right)^{1 / 2}\right\} \\
\leq c b_{0} \sigma_{x}^{2}\left\|\beta_{1}-\beta_{2}\right\|_{2}^{2}\left(\epsilon_{T}^{1 / 2}+\exp \left(-\frac{c^{\prime} T}{\sigma_{x} r}\right)\right)
\end{gathered}
$$

using the assumption that $x_{i}$ is sub-exponential.

Note that the statement of Lemma 8 holds without modification, because the additional factor of $w\left(x_{i}\right)$ vanishes in the Gaussian comparison argument in the proof of the lemma, since $w\left(x_{i}\right) \leq 1$. Furthermore, $\widetilde{f}\left(\beta_{1}, \beta_{2}\right)$ is again a quadratic form in $\beta_{1}-\beta_{2}$, and since $w\left(x_{i}\right) x_{i}$ is bounded and $x_{i}$ is sub-exponential, the quantity $\frac{1}{n} \sum_{i=1}^{n} w\left(x_{i}\right)\left(x_{i}^{T} v\right)^{2}$ is an i.i.d. average of sub-exponential terms with parameter proportional to $b_{0} \sigma_{x}^{2}$. Hence, a version of inequality (87) holds, with $\sigma_{x}^{2}$ replaced by $b_{0} \sigma_{x}^{2}$. Then Lemma 9 follows by an identical peeling argument. Putting together the pieces, we arrive at the desired result.

D.4. Proof of Proposition 4. This is very similar to the proof of Proposition 2. Again using the notation for the Taylor remainder defined in equation (79), we have

$$
\mathcal{T}\left(\beta_{1}, \beta_{2}\right)=\frac{1}{n} \sum_{i=1}^{n} w\left(x_{i}\right) x_{i}^{T}\left(\beta_{1}-\beta_{2}\right)\left\{\ell^{\prime}\left(\left(x_{i}^{T} \beta_{1}-y_{i}\right) w\left(x_{i}\right)\right)-\ell^{\prime}\left(\left(x_{i}^{T} \beta_{2}-y_{i}\right) w\left(x_{i}\right)\right)\right\} .
$$

Defining

$$
A_{i}:=\left\{\left|\epsilon_{i}\right| \leq \frac{T}{2}\right\} \cap\left\{\left|w\left(x_{i}\right) x_{i}^{T}\left(\beta_{1}-\beta_{2}\right)\right| \leq \frac{T}{8 r}\left\|\beta_{1}-\beta_{2}\right\|_{2}\right\} \cap\left\{\left|w\left(x_{i}\right) x_{i}^{T}\left(\beta_{2}-\beta^{*}\right)\right| \leq \frac{T}{4}\right\}
$$

we have that on the event $A_{i}$ and for $\left\|\beta_{1}-\beta^{*}\right\|_{2},\left\|\beta_{2}-\beta^{*}\right\|_{2} \leq r$,

$$
\left|w\left(x_{i}\right)\left(x_{i}^{T} \beta_{2}-y_{i}\right)\right| \leq\left|w\left(x_{i}\right) x_{i}^{T}\left(\beta_{2}-\beta^{*}\right)\right|+\left|w\left(x_{i}\right) \epsilon_{i}\right| \leq\left|w\left(x_{i}\right) x_{i}^{T}\left(\beta_{2}-\beta^{*}\right)\right|+\left|\epsilon_{i}\right| \leq T,
$$

and

$$
\left|w\left(x_{i}\right)\left(x_{i}^{T} \beta_{1}-y_{i}\right)\right| \leq\left|w\left(x_{i}\right) x_{i}^{T}\left(\beta_{1}-\beta_{2}\right)\right|+\left|w\left(x_{i}\right) x_{i}^{T}\left(\beta_{2}-\beta^{*}\right)\right|+\left|w\left(x_{i}\right) \epsilon_{i}\right| \leq \frac{T}{4}+\frac{T}{4}+\frac{T}{2},
$$


using the fact that $w\left(x_{i}\right) \leq 1$. Hence, we have

$$
\begin{aligned}
\mathcal{T}\left(\beta_{1}, \beta_{2}\right) & \geq \alpha_{T} \cdot \frac{1}{n} \sum_{i=1}^{n}\left(w\left(x_{i}\right) x_{i}^{T}\left(\beta_{1}-\beta_{2}\right)\right)^{2} 1_{A_{i}}-\kappa_{2} \cdot \frac{1}{n} \sum_{i=1}^{n}\left(w\left(x_{i}\right) x_{i}^{T}\left(\beta_{1}-\beta_{2}\right)\right)^{2} 1_{A_{i}^{c}} \\
& =\left(\alpha_{T}+\kappa_{2}\right) \cdot \frac{1}{n} \sum_{i=1}^{n}\left(w\left(x_{i}\right) x_{i}^{T}\left(\beta_{1}-\beta_{2}\right)\right)^{2} 1_{A_{i}}-\kappa_{2} \cdot \frac{1}{n} \sum_{i=1}^{n}\left(w\left(x_{i}\right) x_{i}^{T}\left(\beta_{1}-\beta_{2}\right)\right)^{2} .
\end{aligned}
$$

We may define the truncation functions exactly as in the proof of Proposition 2, the only modification being that $x_{i}$ is replaced by $w\left(x_{i}\right) x_{i}$. Furthermore, since $\left|w\left(x_{i}\right) x_{i}^{T} v\right| \leq b_{0}$ for every unit vector $v$, the vector $w\left(x_{i}\right) x_{i}$ is always sub-Gaussian with parameter $b_{0}$, regardless of the distribution of $x_{i}$. It follows that with

$$
\begin{aligned}
f\left(\beta_{1}, \beta_{2}\right) & :=\frac{1}{n} \sum_{i=1}^{n} \varphi_{T\left\|\beta_{1}-\beta_{2}\right\|_{2} / 8 r}\left(w\left(x_{i}\right) x_{i}^{T}\left(\beta_{1}-\beta_{2}\right)\right) \cdot \psi_{T / 2}\left(\epsilon_{i}\right) \cdot \psi_{T / 4}\left(w\left(x_{i}\right) x_{i}^{T}\left(\beta_{2}-\beta^{*}\right)\right), \\
\widetilde{f}\left(\beta_{1}, \beta_{2}\right) & :=\frac{1}{n} \sum_{i=1}^{n}\left(w\left(x_{i}\right) x_{i}^{T}\left(\beta_{1}-\beta_{2}\right)\right)^{2},
\end{aligned}
$$

we arrive at the familiar inequality,

$$
\mathcal{T}\left(\beta_{1}, \beta_{2}\right) \geq\left(\alpha_{T}+\kappa_{2}\right) \cdot f\left(\beta_{1}, \beta_{2}\right)-\kappa_{2} \cdot \widetilde{f}\left(\beta_{1}, \beta_{2}\right) .
$$

The remainder of the proof is identical to the proof of Proposition 2, with $x_{i}$ replaced by $w\left(x_{i}\right) x_{i}$, which is sub-Gaussian with parameter $b_{0}$.

\section{APPENDIX E: PROOFS OF COROLLARIES}

In this Appendix, we provide the proofs of Corollaries 1 and 2.

E.1. Proof of Corollary 1. By Theorem 1, we know that the objective function (2) possesses a stationary point $\widetilde{\beta}$ in the interior of $\underset{\mathbb{B}_{r}}{ }\left(\beta^{*}\right)$ that is a zero-subgradient point of the objective function. We need to show that $\widetilde{\beta}$ is the unique stationary point of the program.

Suppose there exists another stationary point $\widehat{\beta}$ of the objective function. Since $\mathcal{L}_{n}$ is convex, both $\widetilde{\beta}$ and $\widehat{\beta}$ must be global minima of the program (2). Without loss of generality, we may assume that $\widehat{\beta} \in \mathbb{B}_{r}\left(\beta^{*}\right)$, since we may otherwise find a point on the boundary of $\mathbb{B}_{r}\left(\beta^{*}\right)$ lying on the line segment between $\widetilde{\beta}$ and $\widehat{\beta}$, which is also a global minimum of the objective function. It follows from inequality (40) in the proof of Theorem 1 that $\widehat{\nu}=\widehat{\beta}-\beta^{*}$ satisfies the bound

$$
\|\widehat{\nu}\|_{1} \leq 4 \sqrt{k}\|\widehat{\nu}\|_{2}
$$

But this contradicts Assumption 2 when we take $\beta_{1}=\widehat{\beta}$ and $\beta_{2}=\beta^{*}$, since the left-hand expression is 0 and the right-hand side is bounded below

$$
\alpha\left\|\widehat{\beta}-\beta^{*}\right\|_{2}^{2}-\tau \frac{\log p}{n} \cdot k\left\|\widehat{\beta}-\beta^{*}\right\|_{2}^{2} \geq \frac{\alpha}{2}\left\|\widehat{\beta}-\beta^{*}\right\|_{2}^{2}>0
$$


assuming $n \geq \frac{2 \tau}{\alpha} k \log p$. Thus, $\widetilde{\beta}$ must be the unique stationary point of the objective function.

E.2. Proof of Corollary 2. The proof of this corollary is a fairly immediate consequence of Theorem 2 and the following result from He and Shao (2000):

Lemma 11 (Corollary 2.1, He and Shao (2000)). Suppose we have i.i.d. observations from the usual linear regression model

$$
y_{i}=x_{i}^{T} \beta^{*}+\epsilon_{i},
$$

where $\beta^{*} \in \mathbb{R}^{p}$. Suppose

$$
\mathcal{L}_{n}(\beta)=\frac{1}{n} \sum_{i=1}^{n} \ell\left(x_{i}^{T} \beta-y_{i}\right),
$$

and the following conditions are satisfied:

(i) In probability, $0<\lambda_{\min }\left(\frac{X^{T} X}{n}\right)$ and $\lambda_{\max }\left(\frac{X^{T} X}{n}\right)<\infty$.

(ii) $\ell$ is convex and smooth, $\ell^{\prime \prime}$ and $\ell^{\prime \prime \prime}$ are bounded, and $\mathbb{E}\left[\ell^{\prime \prime}\left(\epsilon_{i}\right)\right] \in(0, \infty)$.

(iii) $\max _{1 \leq i \leq n} \frac{\left\|x_{i}\right\|_{2}^{2}}{p}=\mathcal{O}_{P}(1)$ and $\sup _{\|u\|_{2}=\|w\|_{2}=1} \frac{1}{n} \sum_{i=1}^{n}\left|x_{i}^{T} u\right|^{2}\left|x_{i}^{T} w\right|^{2}=\mathcal{O}_{P}(1)$.

Suppose $\mathcal{L}_{n}$ has a unique minimizer given by $\widehat{\beta}$. If $\frac{p \log ^{3} p}{n} \rightarrow 0$, then $\left\|\widehat{\beta}-\beta^{*}\right\|_{2}=\mathcal{O}_{p}\left(\sqrt{\frac{p}{n}}\right)$. If $\frac{p^{2} \log p}{n} \rightarrow 0$, then for any unit vector $v \in \mathbb{R}^{p}$, we have

$$
\frac{\sqrt{n}}{\sigma_{v}} \cdot v^{T}\left(\widehat{\beta}-\beta^{*}\right) \stackrel{d}{\longrightarrow} N(0,1),
$$

where

$$
\sigma_{v}^{2}:=\frac{1}{\mathbb{E}\left[\ell^{\prime \prime}\left(\epsilon_{i}\right)\right] \cdot \mathbb{E}\left[\left(\ell^{\prime}\left(\epsilon_{i}\right)\right)^{2}\right]} \cdot v^{T}\left(\frac{X^{T} X}{n}\right) v
$$

We apply the result to the oracle estimator $\widehat{\beta}_{S}^{\mathcal{O}}$ defined in equation (19), with $k$ taking the place of $p$. Although Lemma 11 requires $\mathcal{L}_{n}$ to be convex, a careful inspection of the proofs in He and Shao (2000) reveals that the results still hold if we restrict our attention to a subset of $\mathbb{R}^{p}$ on which $\mathcal{L}_{n}$ is convex and $\widehat{\beta}$ is the unique minimizer. By Lemma 1 , this is exactly the case over the restricted region $S_{r}$, when $\mathcal{L}_{n}$ satisfies Assumption 2. Furthermore, it is straightforward to check that conditions (i)-(iii) of Lemma 11 under the given assumptions. Note that by Theorem 2.1 in Hsu, Kakade and Zhang (2012), we have

$$
\mathbb{P}\left(\frac{\left\|x_{i}\right\|_{2}^{2}}{k} \geq t\right) \leq c_{1} \exp \left(-c_{2} k\right), \quad \forall i
$$


when the $x_{i}$ 's are sub-Gaussian, implying that

$$
\mathbb{P}\left(\max _{1 \leq i \leq n} \frac{\left\|x_{i}\right\|_{2}^{2}}{k} \geq t\right) \leq n \cdot c_{1} \exp \left(-c_{2} k\right) .
$$

Hence, for $k \geq C \log n$, the right-hand expression is bounded above by $c_{1} \exp \left(-c_{2}^{\prime} k\right)$, and the first part of condition (iii) is satisfied.

We conclude that the desired results hold for the oracle estimator $\widehat{\beta}^{\mathcal{O}}$, and by Theorem 2 , also for $\widetilde{\beta}$.

\section{APPENDIX F: PROOFS OF ADDITIONAL LEMMAS}

In this section, we provide proofs of additional technical lemmas appearing in the body of the paper.

F.1. Proof of Lemma 1. For $\beta_{1}, \beta_{2} \in S_{r}$, we have

$$
\left\|\beta_{1}-\beta_{2}\right\|_{1} \leq \sqrt{k}\left\|\beta_{1}-\beta_{2}\right\|_{2} .
$$

Hence, the RSC condition (10) implies that

$$
\left\langle\nabla \mathcal{L}_{n}\left(\beta_{1}\right)-\nabla \mathcal{L}_{n}\left(\beta_{2}\right), \beta_{1}-\beta_{2}\right\rangle \geq\left(\alpha-\tau \frac{k \log p}{n}\right)\left\|\beta_{1}-\beta_{2}\right\|_{2}^{2}
$$

implying the desired conclusion.

F.2. Proof of Lemma 2. Note that if $X=\left(X_{1}, \ldots, X_{n}\right)$ is a vector of i.i.d. $\alpha$-stable random variables with $\gamma=1$, equation (25) implies that for $w \in \mathbb{R}^{n}$, we have

$$
\mathbb{E}\left[\exp \left(i t \cdot w^{T} X\right)\right]=\exp \left(-\|w\|_{\alpha}^{\alpha}|t|^{\alpha}\right), \quad \forall t>0
$$

where $\|w\|_{\alpha}:=\left(\sum_{i=1}^{n}|w|^{\alpha}\right)^{1 / \alpha}$. Hence, $w^{T} X$ is also $\alpha$-stable, but with the scale parameter $\|w\|_{\alpha}$. Furthermore, if $Z \in \mathbb{R}$ is sub-Gaussian with parameter $\sigma_{z}$, then for $\alpha \in(0,2]$, the random variable $|Z|^{\alpha}$ is sub-exponential with parameter $c \sigma_{z}^{2}$. Indeed, the moments of $|Z|^{\alpha}$ may be bounded as

$$
\mathbb{E}\left[|Z|^{\alpha s}\right]^{1 / s} \leq \mathbb{E}\left[|Z|^{2 s}\right]^{\frac{\alpha}{2 s}} \leq\left(c \sigma_{z} \sqrt{s}\right)^{\alpha} \leq c^{\prime} \sigma_{z}^{2} s
$$

where the first inequality comes from Hölder's inequality and the second inequality follows because $Z$ is sub-Gaussian (Vershynin, 2012). Hence, $Z^{\alpha}$ is sub-exponential. Consequently, for any $1 \leq j \leq p$, the quantity

$$
\left\|\frac{X e_{j}}{n^{1 / \alpha}}\right\|_{\alpha}^{\alpha}=\frac{1}{n}\left\|X e_{j}\right\|_{\alpha}^{\alpha}=\frac{1}{n} \sum_{i=1}^{n}\left|x_{i j}\right|^{\alpha}
$$


exhibits sub-exponential concentration to $\mathbb{E}\left[\left|X_{i j}\right|^{\alpha}\right]$.

In the context of ordinary least squares regression with the Lasso, note that for an arbitrary $1 \leq j \leq p$, we have

$$
\mathbb{P}\left(\left\|\frac{X^{T} \epsilon}{n}\right\|_{\infty} \geq \lambda\right)=\mathbb{P}\left(\left\|\frac{X^{T} \epsilon}{n^{1 / \alpha}}\right\|_{\infty} \geq n^{1-1 / \alpha} \lambda\right) \geq \mathbb{P}\left(\left|\frac{e_{j}^{T} X^{T} \epsilon}{n^{1 / \alpha}}\right| \geq n^{1-1 / \alpha} \lambda\right) .
$$

Since $\left|\frac{e_{j}^{T} X \epsilon}{n^{1 / \alpha}}\right|$ is $\alpha$-stable with scale parameter $\Theta\left(\mathbb{E}\left[\left|X_{i j}\right|^{\alpha}\right]\right)$, by the above discussion, the right-hand expression in inequality (103) is bounded below by a constant $c_{\alpha}$ whenever $n^{1-1 / \alpha} \lambda \rightarrow 0$. In particular, this is the case when $\alpha<2$. Hence, we conclude that the bound

$$
\left\|\frac{X^{T} \epsilon}{n}\right\|_{\infty} \precsim \sqrt{\frac{\log p}{n}}
$$

does not hold w.h.p. when the entries of $\epsilon$ are drawn from an $\alpha$-stable distribution with $\alpha<2$.

Finally, recall that if $\widehat{\beta}$ is a global solution for the Lasso and $\lambda \succsim\left\|\frac{X^{T} \epsilon}{n}\right\|_{\infty}$, we have the $\ell_{2}$-error bound

$$
\left\|\widehat{\beta}-\beta^{*}\right\|_{2} \leq c \sqrt{k} \cdot \max \left\{\lambda,\left\|\frac{X^{T} \epsilon}{n}\right\|_{\infty}\right\}
$$

with high probability (Bickel, Ritov and Tsybakov, 2009). This establishes the inconsistency of the Lasso estimator.

\section{REFERENCES}

AdAmczak, R. and WolfF, P. (2014). Concentration inequalities for non-Lipschitz functions with bounded derivatives of higher order. Probability Theory and Related Fields 1-56.

Agarwal, A., Negahban, S. and Wainwright, M. J. (2012). Fast global convergence of gradient methods for high-dimensional statistical recovery. Annals of Statistics 40 2452-2482.

Alfons, A., Croux, C. and Gelper, S. (2013). Sparse least trimmed squares regression for analyzing high-dimensional large data sets. Annals of Applied Statistics 7 226-248.

Avella Medina, M. A. (2014). Influence functions for penalized $M$-estimators Technical Report, University of Geneva. Available at http://archive-ouverte.unige.ch/unige: 35319.

BAI, Z. D. and Wu, Y. (1997). General M-estimation. Journal of Multivariate Analysis 63 119-135.

Bean, D., Bickel, P. J., El Karoui, N. and Yu, B. (2013). Optimal $M$-estimation in high-dimensional regression. Proceedings of the National Academy of Sciences 110 14563-14568.

Bertsekas, D. P. (1999). Nonlinear Programming. Athena Scientific, Belmont, MA.

Bickel, P. J. (1975). One-step Huber estimates in the linear model. Journal of the American Statistical Association $\mathbf{7 0}$ 428-434.

Bickel, P. J., Ritov, Y. and Tsybakov, A. (2009). Simultaneous analysis of Lasso and Dantzig selector. Annals of Statistics 37 1705-1732.

Box, G. E. P. (1953). Non-normality and tests on variances. Biometrika 40 318-335.

Bradic, J., FAn, J. and WAng, W. (2011). Penalized composite quasi-likelihood for ultrahigh dimensional variable selection. Journal of the Royal Statistical Society: Series B (Statistical Methodology) 73 325-349.

Clarke, F. H. (1983). Optimization and Nonsmooth Analysis. Wiley-Interscience, New York. 
Donoho, D. and Huber, P. (1983). The notion of breakdown point. In A Festschrift for Erich L. Lehmann (P. J. Bickel, K. A. Doksum and J. L. Hodges Jr., eds.) 157-184. Wadsworth, Belmont, CA.

Donoho, D. and Montanari, A. (2013). High dimensional robust $M$-estimation: Asymptotic variance via approximate message passing. Probability Theory and Related Fields 1-35.

El Karoui, N., Bean, D., Bickel, P. J., Lim, C. and Yu, B. (2013). On robust regression with highdimensional predictors. Proceedings of the National Academy of Sciences 110 14557-14562.

FAN, J. and LI, R. (2001). Variable selection via nonconcave penalized likelihood and its oracle properties. Journal of the American Statistical Association 96 1348-1360.

FAn, J., LI, Q. and WANG, Y. (2014). Robust estimation of high-dimensional mean regression. ArXiv e-prints. Available at http://arxiv.org/abs/1410.2150.

FAn, J. and Peng, H. (2004). Nonconcave penalized likelihood with a diverging number of parameters. Annals of Statistics 32 928-961.

Fletcher, R. and Watson, G. A. (1980). First and second order conditions for a class of nondifferentiable optimization problems. Mathematical Programming 18 291-307.

Freedman, D. A. and Diaconis, P. (1982). On inconsistent M-estimators. Annals of Statistics $10454-$ 461.

Friedman, J., Hastie, T. and Tibshirani, R. (2008). Sparse inverse covariance estimation with the graphical Lasso. Biostatistics 9 432-441.

Godambe, V. P. (1960). An optimum property of regular maximum likelihood estimation. Annals of Mathematical Statistics 31 1208-1211.

HAMPEL, F. R. (1968). Contributions to the theory of robust estimation PhD thesis, University of California at Berkeley.

Hampel, F. R., Ronchetti, E. M., Rousseeuw, P. J. and Stahel, W. A. (2011). Robust Statistics: The Approach Based on Influence Functions. Wiley Series in Probability and Statistics. Wiley.

He, X. and ShaO, Q. M. (1996). A general Bahadur representation of $M$-estimators and its application to linear regression with nonstochastic designs. Annals of Statistics 24 2608-2630.

He, X. and Shao, Q. M. (2000). On parameters of increasing dimensions. Journal of Multivariate Analysis 73 120-135.

HiLl, R. W. (1977). Robust regression when there are outliers in the carriers. Harvard University PhD dissertation.

Hsu, D., KAKAde, S. and ZhAng, T. (2012). A tail inequality for quadratic forms of subgaussian random vectors. Electronic Communications in Probability 17 no. 52, 1-6.

Huber, P. J. (1964). Robust estimation of a location parameter. Annals of Mathematical Statistics 35 73-101.

Huber, P. J. (1973). Robust regression: Asymptotics, conjectures and Monte Carlo. Annals of Statistics 1 799-821.

Huber, P. J. (1981). Robust Statistics. Wiley New York.

Ledoux, M. and Talagrand, M. (1991). Probability in Banach Spaces: Isoperimetry and Processes. Springer-Verlag, New York, NY.

Lehmann, E. L. and Casella, G. (1998). Theory of Point Estimation. Springer Verlag.

Li, G., Peng, H. and Zhu, L. (2011). Nonconcave penalized $M$-estimation with a diverging number of parameters. Statistica Sinica 21 391-419.

Loh, P. and Wainwright, M. J. (2012). High-dimensional regression with noisy and missing data: Provable guarantees with non-convexity. Annals of Statistics 40 1637-1664.

Loh, P. and Wainwright, M. J. (2014). Support recovery without incoherence: A case for nonconvex regularization. arXiv e-prints. Available at http://arxiv.org/abs/1412.5632.

Loh, P. and Wainwright, M. J. (2015). Regularized $M$-estimators with nonconvexity: Statistical and algorithmic theory for local optima. Journal of Machine Learning Research 16 559-616.

Lozano, A. C. and Meinshausen, N. (2013). Minimum distance estimation for robust high-dimensional regression. ArXiv e-prints. Available at http://arxiv.org/abs/1307.3227. 
Mallows, C. L. (1975). On some topics in robustness. Unpublished memorandum. Bell Telephone Laboratories, Murray Hill, NJ.

Mammen, E. (1989). Asymptotics with increasing dimension for robust regression with applications to the bootstrap. Annals of Statistics 17 382-400.

Maronna, R., Bustos, O. and Yohai, V. (1979). Bias- and efficiency-robustness of general $M$-estimators for regression with random carriers. In Smoothing Techniques for Curve Estimation, (T. Gasser and M. Rosenblatt, eds.). Lecture Notes in Mathematics 757 91-116. Springer Berlin Heidelberg.

Maronna, R. A., Martin, R. D. and Yohai, V. J. (2006). Robust Statistics: Theory and Methods. J. Wiley.

Maronna, R. A. and Yohai, V. J. (1981). Asymptotic behavior of general $M$-estimates for regression and scale with random carriers. Zeitschrift für Wahrscheinlichkeitstheorie und Verwandte Gebiete 58 7-20.

Mendelson, S. (2014). Learning without concentration for general loss functions. ArXiv e-prints. Available at http://arxiv.org/abs/1410.3192.

Merrill, H. M. and Schweppe, F. C. (1971). Bad data suppression in power system static state estimation. IEEE Transactions on Power Apparatus and Systems PAS-90 2718-2725.

Negahban, S., Ravikumar, P., Wainwright, M. J. and Yu, B. (2012). A unified framework for highdimensional analysis of $M$-estimators with decomposable regularizers. Statistical Science 27 538-557. See arXiv version for lemma/propositions cited here.

Nesterov, Y. (2007). Gradient methods for minimizing composite objective function CORE Discussion Papers No. 2007076, Universit Catholique de Louvain, Center for Operations Research and Econometrics (CORE).

Nolan, J. P. (2015). Stable Distributions - Models for Heavy Tailed Data. Birkhauser, Boston. In progress, Chapter 1 online at http://academic2.american.edu/ jpnolan.

Öllerer, V., Croux, C. and Alfons, A. (2014). The influence function of penalized regression estimators. Statistics.

Portnoy, S. (1985). Asymptotic behavior of $M$ estimators of $p$ regression parameters when $p^{2} / n$ is large; II. Normal approximation. Annals of Statistics 13 1403-1417.

Raskutti, G., Wainwright, M. J. and Yu, B. (2010). Restricted eigenvalue properties for correlated Gaussian designs. Journal of Machine Learning Research 11 2241-2259.

Ravikumar, P., Wainwright, M. J. and Lafferty, J. D. (2010). High-dimensional Ising model selection using $\ell_{1}$-regularized logistic regression. Annals of Statistics 381287.

Rousseeuw, P. J. (1981). A new infinitesimal approach to robust estimation. Zeitschrift für Wahrscheinlichkeitstheorie und Verwandte Gebiete 56 127-132.

Rousseeuw, P. J. and Leroy, A. M. (2005). Robust Regression and Outlier Detection. Wiley Series in Probability and Statistics. Wiley.

Rudelson, M. and Zhou, S. (2013). Reconstruction from anisotropic random measurements. IEEE Transactions on Information Theory 59 3434-3447.

Shevlyakov, G., Morgenthaler, S. and Shurygin, A. (2008). Redescending M-estimators. J. Stat. Plann. Inference 138 2906-2917.

Simpson, D. G., Ruppert, D. and Carroll, R. J. (1992). On one-step GM estimates and stability of inferences in linear regression. Journal of the American Statistical Association 87 439-450.

Tibshirani, R. (1996). Regression shrinkage and selection via the Lasso. Journal of the Royal Statistical Society, Series B 58 267-288.

Tukey, J. W. (1960). A survey of sampling from contaminated distributions. In Contributions to probability and statistics: Essays in Honor of Harold Hotelling (I. Olkin, S. G. Ghurye, W. Hoeffding, W. G. Madow and H. B. Mann, eds.) 448-485. Stanford University Press.

VERSHYNin, R. (2012). Introduction to the non-asymptotic analysis of random matrices. Compressed Sensing 210-268.

Wainwright, M. J. (2009). Sharp thresholds for high-dimensional and noisy sparsity recovery using $\ell_{1}$ constrained quadratic programming (Lasso). IEEE Transactions on Information Theory 55 2183-2202. 
WANG, L. (2013). The $L 1$ penalized LAD estimator for high dimensional linear regression. Journal of Multivariate Analysis 120 135-151.

WANG, H., LI, G. and JiAng, G. (2007). Robust regression shrinkage and consistent variable selection through the LAD-Lasso. Journal of Business 83 Economic Statistics 25 347-355.

Wang, X., JiAng, Y., Huang, M. and Zhang, H. (2013). Robust variable selection with exponential squared loss. Journal of the American Statistical Association 108 632-643.

Welsh, A. H. and Ronchetti, E. (2002). A journey in single steps: Robust one-step $M$-estimation. Journal of Statistical Planning and Inference 103 287-310.

Yoнai, V. J. (1987). High breakdown-point and high efficiency robust estimates for regression. Annals of Statistics 15 642-656.

Yohai, V. J. and Maronna, R. A. (1979). Asymptotic behavior of $M$-estimators for the linear model. Annals of Statistics 7 258-268.

Zhang, T. (2010a). Analysis of multi-stage convex relaxation for sparse regularization. Journal of Machine Learning Research 11 1081-1107.

ZhANG, C. H. (2010b). Nearly unbiased variable selection under minimax concave penalty. Annals of Statistics 38 894-942.

Departments of ECE \& Statistics

1415 Engineering Drive

MAdison, Wisconsin 53706

USA

E-MAIL: loh@ece.wisc.edu 